\title{
Two-fluid hydrodynamics of cold atomic bosons under influence of the quantum fluctuations at non-zero temperatures
}

\author{
Pavel A. Andreev ${ }^{*}$ \\ Faculty of physics, Lomonosov Moscow State University, Moscow, Russian Federation, 119991. and \\ Peoples Friendship University of Russia (RUDN University), \\ 6 Miklukho-Maklaya Street, Moscow, 117198, Russian Federation
}

(Dated: January 29, 2021)

\begin{abstract}
Ultracold Bose atoms is the physical system, where the quantum and nonlinear phenomena play crucial role. Ultracold bosons are considered at the small finite temperatures. Bosons are considered as two different fluids: Bose-Einstein condensate and normal fluid (the thermal component). An extended hydrodynamic model is obtained for both fluids, where the pressure evolution equations and the pressure flux third rank tensor evolution equations are considered along with the continuity and Euler equations. It is found that the pressure evolution equation contains zero contribution of the short-range interaction. The pressure flux evolution equation contains the interaction which gives the quantum fluctuations in the zero temperature limit. Here, we obtain its generalization for the finite temperature. The contribution of interaction in the pressure flux evolution equation which goes to zero in the zero temperature limit is found. The model is obtained via the straightforward derivation from the microscopic many-particle Schrodinger equation in the coordinate representation.
\end{abstract}

\section{INTRODUCTION}

Small temperature bosons are studied in terms of twofluid hydrodynamics consisting of the Bose-Einstein condensate (BEC) and normal fluid [1]. Each fluid is considered in terms of two hydrodynamic equations: the continuity and Euler equations. It is assumed that the BEC can be completely described by the concentration and velocity field, or, in other terms, by the Gross-Pitaevskii equation [1], since BEC is the collection of particles in the single quantum state. However, the normal fluid model requires an truncation of the set of hydrodynamic equations. The pressure of normal fluid existing in the Euler equation for the normal fluid is an independent function. Equation for the pressure evolution provides an expression for the pressure perturbations via the perturbations of other functions. Application of the equation of state for pressure makes the model more simple, but equation of state for pressure leads to the less accurate model.

Moreover, the kinetic pressure in the Euler equation for the BEC is usually chosen to be equal to zero [2], [3]. Since the kinetic pressure is related to the occupation of the excited states However, there is the nonzero part caused by the quantum fluctuations [4]. The pressure evolution equation of the weakly interacting bosons contains no interaction, but next equation in the chain of the quantum hydrodynamic equations (the equation for the pressure flux third rank tensor) contains the interaction causing the depletion of the BECs at the zero temperature. Therefore, it is necessary to consider the pressure flux evolution equation both for the BEC and for the normal fluid at the analysis of the small temperature influence.

*Electronic address: andreevpa@physics.msu.ru
The quantum depletion of the BECs is the appearance of the bosons in the excited states while system is kept at the zero temperature. So, some energy of the collective motion is transferred to the individual motion of a portion of particles. It is caused by the quantum fluctuations related to the interparticle interaction. The quantum fluctuations are considered in literature for a long time. Mostly, their theoretical analysis is based on the Bogoliubov-de Gennes approach [5], [6], [7], [8]. The quantum fluctuations in BECs are studied experimentally as well [9], 10], [1]. This method is generalized for the dipolar BECs, where the quantum fluctuations plays crucial role at the description of the dipolar BECs of lantanoids. The dipolar lantanoid BECs reveal the large scale instability causing the splitting of the cloud of atoms on the number of macroscopic drops. This highly nonlinear phenomena is called the quantum droplet formation [12 31]. Therefore, reassemble of bosons in smaller compact groups causes the stabilization of the system. These studies give the motivation for the study of quantum fluctuations. However, here we restrict our analysis with the short-range interaction only and no dipole-dipole interaction is discussed. Moreover, the influence of the finite temperature is the necessary part of complete model of these phenomena.

Fundamental feature of the collective dynamics in the spectrum of the sound waves. Two distinct sound velocities exist in finite temperature ultracold Bose gas [1], 32]. The two-fluid model shows that the slower mode (second sound) is associated with the BEC component, while the faster mode (first sound) is associated with the thermal component. Generalized expressions for the speeds of sounds are obtained within developed model.

Derivation of two-fluid hydrodynamics for the finite temperature bosons in the limit of small temperature, where the large fraction of the bosons is located in the $\mathrm{BEC}$ state is given from the microscopic motion in accor- 
dance with the quantum hydrodynamic method [2], [3], [33], 34]. The microscopic dynamics is described by the Schrodinger equation in the coordinate representation. Collection of the macroscopic functions is presented to describe the collective effects in ultracold bosons. The last includes the concentration of particles, the velocity field and the pressure tensor. The derivation of basic equations is made for all bosons distributed on the lower energy level and the excited levels as the single fluid. The decomposition on two fluids is made on the microscopic scale. After general structure of equations is obtained for the arbitrary temperature and arbitrary strength of interaction, an approximate calculation of functions presenting the interaction is made for the regime of shortrange interaction. Hence, the small parameter related to the small area of interaction potential is used. It gives a specification for general model, but also the first order contribution on the small parameter is applied. The further truncation is made at calculation of the interaction terms for weak interaction and small temperatures.

This paper is organized as follows. In Sec. II major steps of derivation of hydrodynamic equations from the Schrodinger equation are demonstrated, where the pressure evolution equation (the quantum Bohm potential evolution equation) and the third rank tensor evolution equation are obtained along with the continuity and $\mathrm{Eu}-$ ler equations. In Sec. III calculation of interaction in the Euler equation, the pressure evolution equation, and the pressure flux evolution equation is demonstrated. In Sec. IV presents the suggested version of the extended two-fluid quantum hydrodynamic model for the ultracold finite temperature bosons. In Sec. V the limiting regime of derived model for the BEC is obtained under influence of the quantum fluctuations at the zero temperature. In Sec. VI a brief summary of obtained results is presented.

\section{ON DERIVATION OF HYDRODYNAMIC EQUATIONS FROM MICROSCOPIC QUANTUM DYNAMICS}

\section{A. Basic definitions of quantum hydrodynamics and the Euler equation derivation}

On the microscopic level we do not have notion of temperature. Hence we consider system of interacting bosons governed by the Schrodinger equation $\imath \hbar \partial_{t} \Psi=\hat{H} \Psi$ with the following Hamiltonian

$$
\hat{H}=\sum_{i=1}^{N}\left(\frac{\hat{\mathbf{p}}_{i}^{2}}{2 m_{i}}+V_{e x t}\left(\mathbf{r}_{i}, t\right)\right)+\frac{1}{2} \sum_{i, j \neq i} U\left(\mathbf{r}_{i}-\mathbf{r}_{j}\right),
$$

where $m_{i}$ is the mass of $\mathrm{i}$-th particle, $\hat{\mathbf{p}}_{i}=-\imath \hbar \nabla_{i}$ is the momentum of i-th particle. The last term in the Hamiltonian (11) is the boson-boson interaction $U_{i j}$. We do not specify the form of interaction. However, the derivation presented below employs that the interaction has finite value on the small distances between particles and shows fast decay at the increase of the interparticle distance. Definitely, no distinguishing between bosons in the BEC state and bosons in other states is made at this stage. Separation of all bosons on two subsystems is made in terms of collective variables.

Hydrodynamic model usually made for each species of particles. If we consider a single species then all masses equal to each other.

Distribution of particles in a trap, waves, solitons, oscillations of form of trapped particles are described by the concentration of particles. Concentration is an essential macroscopic function both for classical and for quantum fluids. The module of the macroscopic wave function in the Gross-Pitaevskii equation gives the square root of concentration of bosons in the BEC state. Therefore, we start the derivation of quantum hydrodynamic equations from the definition of concentration. The quantum mechanics is based on notion of point-like objects in spite the wave nature of quantum objects. So, the eigenfunction of the coordinate operator in the coordinate representation is the delta function $\hat{x} \psi_{x^{\prime}}(x)=x^{\prime} \psi_{x^{\prime}}(x)$, where normalized wave function is $\psi_{x^{\prime}}(x)=\delta\left(x-x^{\prime}\right)$. Obviously, the operation of concentration in the coordinate representation of quantum mechanics is the sum of delta functions $\hat{n}=\sum_{i=1}^{N} \delta\left(\mathbf{r}-\mathbf{r}_{i}\right)$. Moreover, it is supported by the general rule for quantization. We need to take corresponding classical function.

Transition to description of the collective motion of bosons is made via introduction of the concentration [2], 33]:

$$
n=\int d R \sum_{i=1}^{N} \delta\left(\mathbf{r}-\mathbf{r}_{i}\right) \Psi^{*}(R, t) \Psi(R, t),
$$

which is the first collective variable in our model. Other collective variables appear during the derivation. Equation (2) contains the following notations $d R=\prod_{i=1}^{N} d \mathbf{r}_{i}$ is the element of volume in $3 N$ dimensional configurational space, with $N$ is the number of bosons. Concentration (22) is the sum of partial concentrations $n=n_{n}+n_{b}$ describing the distribution of BEC $n_{b}$ and normal fluid $n_{n}$ in the coordinate space.

The equation for evolution of concentration (2) can be obtained by acting by time derivative on function (2). The time derivative acts on the wave functions under the integral while the time derivatives of the wave function are taken from the Schrodinger equation. Obtain the continuity equation for concentration (2) after straightforward calculations

$$
\partial_{t} n+\nabla \cdot \mathbf{j}=0
$$

where the new collective function called the current appears as the following integral of the wave function

$$
\mathbf{j}(\mathbf{r}, t)=\int d R \sum_{i=1}^{N} \delta\left(\mathbf{r}-\mathbf{r}_{i}\right) \times
$$




$$
\times \frac{1}{2 m_{i}}\left(\Psi^{*}(R, t) \hat{\mathbf{p}}_{i} \Psi(R, t)+\text { c.c. }\right),
$$

with c.c. is the complex conjugation.

Both introduced collective functions $n(\mathbf{r}, t)$ and $\mathbf{j}(\mathbf{r}, t)$ are quadratic forms of the wave function. Each of them can be splitted on two parts related to the BEC and normal fluid. Hence we have $n=n_{n}+n_{b}$ and $\mathbf{j}=\mathbf{j}_{n}+\mathbf{j}_{b}$. No microscopic definitions are introduced for the partial functions $n_{n}, n_{b}, \mathbf{j}_{n}$, and $\mathbf{j}_{b}$. Therefore, the continuity equation (3) splits on two partial continuity equations

$$
\partial_{t} n_{a}+\nabla \cdot \mathbf{j}_{a}=0
$$

where subindex $a$ stands for $b$ and $n$.

Continue the derivation of hydrodynamic equations and consider the time evolution of the particle current (41). Act by time derivative on function $\mathbf{j}$ (4) and use the Schrodinger equation with Hamiltonian (11). It leads to the general form of the current evolution equation

$$
\partial_{t} j^{\alpha}+\partial_{\beta} \Pi^{\alpha \beta}=-\frac{1}{m} n \partial_{\alpha} V_{e x t}+\frac{1}{m} F_{i n t}^{\alpha},
$$

where

$$
\begin{gathered}
\Pi^{\alpha \beta}=\int d R \sum_{i=1}^{N} \delta\left(\mathbf{r}-\mathbf{r}_{i}\right) \frac{1}{4 m^{2}}\left[\Psi^{*}(R, t) \hat{p}_{i}^{\alpha} \hat{p}_{i}^{\beta} \Psi(R, t)\right. \\
\left.+\hat{p}_{i}^{\alpha *} \Psi^{*}(R, t) \hat{p}_{i}^{\beta} \Psi(R, t)+\text { c.c. }\right]
\end{gathered}
$$

is the momentum flux, and

$$
F_{\text {int }}^{\alpha}=-\int\left(\partial^{\alpha} U\left(\mathbf{r}-\mathbf{r}^{\prime}\right)\right) n_{2}\left(\mathbf{r}, \mathbf{r}^{\prime}, t\right) d \mathbf{r}^{\prime},
$$

with the two-particle concentration

$$
\begin{gathered}
n_{2}\left(\mathbf{r}, \mathbf{r}^{\prime}, t\right) \\
=\int d R \sum_{i, j=1, j \neq i}^{N} \delta\left(\mathbf{r}-\mathbf{r}_{i}\right) \delta\left(\mathbf{r}^{\prime}-\mathbf{r}_{j}\right) \Psi^{*}(R, t) \Psi(R, t) .
\end{gathered}
$$

It is necessary to split equation (6) on two equations for each subsystem of bosons. In current form equation (6) consist of superposition of functions which are quadratic forms of the wave function. Hence, each term can be splitted on two parts and we find two similar equations for the currents

$$
\partial_{t} j_{a}^{\alpha}+\partial_{\beta} \Pi_{a}^{\alpha \beta}=-\frac{1}{m} n_{a} \partial_{\alpha} V_{e x t}+\frac{1}{m} F_{a, i n t}^{\alpha} .
$$

The first and third terms are proportional to the concentration and the current. therefore, they require no comments. Nontrivial difference between two current evolution equation appears at further analysis of the momentum flux $\Pi^{\alpha \beta}$ and the interaction $F_{i n t}^{\alpha}$. However, we point out some difference which appear for the momentum flux
$\Pi^{\alpha \beta}$. Its structure is obtained in many papers (see for instance [2] after equation (52), [35] equation (24))

$$
\Pi^{\alpha \beta}=n v^{\alpha} v^{\beta}+p^{\alpha \beta}+T^{\alpha \beta},
$$

where $p^{\alpha \beta}$ is the pressure tensor, and $T^{\alpha \beta}$ is the tensor giving the quantum Bohm potential, its approximate form can be written in the following form

$$
T_{0}^{\alpha \beta}=-\frac{\hbar^{2}}{4 m^{2}}\left[\partial_{\alpha} \partial_{\beta} n-\frac{\partial_{\alpha} n \cdot \partial_{\beta} n}{n}\right] .
$$

Tensor $T_{0}^{\alpha \beta}$ (12) is obtained for noninteracting particles located in the single quantum state.

Basically, the pressure tensor $p^{\alpha \beta}$ is defined via the wave function $\Psi(R, t)$. However, it requires some manipulations with the wave function and introduction of a number of intermediate function. Hence we do not present its explicit form. Nevertheless, the pressure tensor is related to the distribution of bosons on quantum states with energies above $E_{m i n}$. Therefore, for bosons in the BEC state we have $p_{B}^{\alpha \beta}=0$ if no quantum fluctuations are considered and

$$
\Pi_{B}^{\alpha \beta}=n_{B} v_{B}^{\alpha} v_{B}^{\beta}+T_{B}^{\alpha \beta}+p_{q f}^{\alpha \beta},
$$

where $T_{B}^{\alpha \beta}$ is the function of $n_{B}$ (12) if there is no interaction. Distribution of particles on different quantum states does not allow to get full expression (12), but the first term. However, it can be used as an equation of state for noninteracting limit. The normal fluid bosons have nonzero pressure $p_{n}^{\alpha \beta} \neq 0$. Hence, all terms in presentation (11) exists in this regime. Expression (12) appears for bosons in the single state in the absence of interaction. Hence, it is an approximate expression for the weakly interacting bosons being in the BEC state. It is even less accurate for normal fluid bosons, but we use it as an equation of state for the quantum part of the momentum flux.

\section{B. The pressure evolution equation}

Extending the set of hydrodynamic equations we can derive the equation for the momentum flux evolution. It can be expected that this equation brings extra information for the normal fluid bosons only. However, the quantum fluctuations give contribution in the evolution of the kinetic pressure of BECs in the limit of zero temperature via the divergence of the third rank tensor. If the temperature is nonzero we have two partial kinetic pressures for the BEC and for the normal fluid. Consider the time evolution of the momentum flux (77) using the Schrodinger equation with Hamiltonian (1).

It gives to the following expression:

$$
\partial_{t} \Pi^{\alpha \beta}=\frac{\imath}{\hbar} \int d R \sum_{i=1}^{N} \delta\left(\mathbf{r}-\mathbf{r}_{i}\right) \frac{1}{4 m^{2}}\left[\hat{H}^{*} \Psi^{*}(R, t) \hat{p}_{i}^{\alpha} \hat{p}_{i}^{\beta} \Psi(R, t)\right.
$$




$$
\begin{gathered}
-\Psi^{*}(R, t) \hat{p}_{i}^{\alpha} \hat{p}_{i}^{\beta} \hat{H} \Psi(R, t)+\hat{p}_{i}^{\alpha *} \hat{H}^{*} \Psi^{*}(R, t) \hat{p}_{i}^{\beta} \Psi(R, t) \\
\left.-\hat{p}_{i}^{\alpha *} \Psi^{*}(R, t) \hat{p}_{i}^{\beta} \hat{H} \Psi(R, t)-c . c .\right]
\end{gathered}
$$

The part of the presented terms contains the Hamiltonian $\hat{H}$ under action of the momentum operators. We permute the Hamiltonian $\hat{H}$ and the operators acting on it. Hence, the result of permutation presented by terms where no operators act on the Hamiltonian $\hat{H}$. However, the terms containing the corresponding commutators appear. Therefore, all terms are combined in two groups:

$$
\begin{gathered}
\partial_{t} \Pi^{\alpha \beta}=\frac{\imath}{\hbar} \int d R \sum_{i=1}^{N} \delta\left(\mathbf{r}-\mathbf{r}_{i}\right) \frac{1}{4 m^{2}}\left[\hat{H}^{*} \Psi^{*} \cdot \hat{p}_{i}^{\alpha} \hat{p}_{i}^{\beta} \Psi\right. \\
\left.-\Psi^{*} \hat{H} \hat{p}_{i}^{\alpha} \hat{p}_{i}^{\beta} \Psi+\hat{H}^{*} \hat{p}_{i}^{\alpha *} \Psi^{*} \cdot \hat{p}_{i}^{\beta} \Psi-\hat{p}_{i}^{\alpha *} \Psi^{*} \cdot \hat{H} \hat{p}_{i}^{\beta} \Psi-c . c .\right] \\
+\frac{\imath}{\hbar} \int d R \sum_{i=1}^{N} \delta\left(\mathbf{r}-\mathbf{r}_{i}\right) \frac{1}{4 m^{2}}\left[-\Psi^{*}\left[\hat{p}_{i}^{\alpha} \hat{p}_{i}^{\beta}, \hat{H}\right] \Psi\right. \\
\left.+\left[\hat{p}_{i}^{\alpha *}, \hat{H}^{*}\right] \Psi^{*} \cdot \hat{p}_{i}^{\beta} \Psi-\hat{p}_{i}^{\alpha *} \Psi^{*} \cdot\left[\hat{p}_{i}^{\beta}, \hat{H}\right] \Psi-c . c .\right]
\end{gathered}
$$

The first group of terms in expression (15) gives the divergence of flux of tensor $\Pi^{\alpha \beta}$. The second group of terms contains the commutators. This group leads to the contribution of interaction in the momentum flux evolution.

It gives the momentum flux evolution equation

$$
\begin{gathered}
\partial_{t} \Pi^{\alpha \beta}+\partial_{\gamma} M^{\alpha \beta \gamma}=-\frac{1}{m} j^{\beta} \partial_{\alpha} V_{e x t} \\
-\frac{1}{m} j^{\alpha} \partial_{\beta} V_{e x t}+\frac{1}{m}\left(F^{\alpha \beta}+F^{\beta \alpha}\right),
\end{gathered}
$$

where the momentum flux is the full flux of all bosons $\Pi^{\alpha \beta}=\Pi_{n}^{\alpha \beta}+\Pi_{b}^{\alpha \beta}$, the splitting on two subspecies is to be made later,

$$
F^{\alpha \beta}=-\int\left[\partial^{\alpha} U\left(\mathbf{r}-\mathbf{r}^{\prime}\right)\right] j_{2}^{\beta}\left(\mathbf{r}, \mathbf{r}^{\prime}, t\right) d \mathbf{r}^{\prime}
$$

is the force tensor field,

$$
\begin{gathered}
M^{\alpha \beta \gamma}=\int d R \sum_{i=1}^{N} \delta\left(\mathbf{r}-\mathbf{r}_{i}\right) \frac{1}{8 m_{i}^{3}}\left[\Psi^{*}(R, t) \hat{p}_{i}^{\alpha} \hat{p}_{i}^{\beta} \hat{p}_{i}^{\gamma} \Psi(R, t)\right. \\
+\hat{p}_{i}^{\alpha *} \Psi^{*}(R, t) \hat{p}_{i}^{\beta} \hat{p}_{i}^{\gamma} \Psi(R, t)+\hat{p}_{i}^{\alpha *} \hat{p}_{i}^{\gamma *} \Psi^{*}(R, t) \hat{p}_{i}^{\beta} \Psi(R, t) \\
\left.+\hat{p}_{i}^{\gamma *} \Psi^{*}(R, t) \hat{p}_{i}^{\alpha} \hat{p}_{i}^{\beta} \Psi(R, t)+\text { c.c. }\right]
\end{gathered}
$$

is the current (flux) of the momentum flux, and

$$
\mathbf{j}_{2}\left(\mathbf{r}, \mathbf{r}^{\prime}, t\right)=\int d R \sum_{i, j \neq i} \delta\left(\mathbf{r}-\mathbf{r}_{i}\right) \delta\left(\mathbf{r}^{\prime}-\mathbf{r}_{j}\right) \times
$$

$$
\times \frac{1}{2 m_{i}}\left[\Psi^{*}(R, t) \hat{\mathbf{p}}_{i} \Psi(R, t)+c . c .\right]
$$

is the two-particle current-concentration function.

If quantum correlations are dropped function $j_{2}^{\alpha}\left(\mathbf{r}, \mathbf{r}^{\prime}, t\right)$ splits on product of the current $j^{\alpha}(\mathbf{r}, t)$ and the concentration $n\left(\mathbf{r}^{\prime}, t\right)$. Interaction in the momentum flux evolution equation (16) is presented by symmetrized combinations of tensors $F^{\alpha \beta}$, which is the flux or current of force.

Partial momentum flux equations appear as

$$
\begin{gathered}
\partial_{t} \Pi_{a}^{\alpha \beta}+\partial_{\gamma} M_{a}^{\alpha \beta \gamma}=-\frac{1}{m} j_{a}^{\beta} \partial_{\alpha} V_{e x t} \\
-\frac{1}{m} j_{a}^{\alpha} \partial_{\beta} V_{e x t}+\frac{1}{m}\left(F_{a}^{\alpha \beta}+F_{a}^{\beta \alpha}\right),
\end{gathered}
$$

where $M^{\alpha \beta \gamma}=M_{B}^{\alpha \beta \gamma}+M_{n}^{\alpha \beta \gamma}$, with

$$
\begin{gathered}
M_{a}^{\alpha \beta \gamma}=n_{a} v_{a}^{\alpha} v_{a}^{\beta} v_{a}^{\gamma}+v_{a}^{\alpha}\left(p_{a}^{\beta \gamma}+T_{a}^{\beta \gamma}\right)+v_{a}^{\beta}\left(p_{a}^{\alpha \gamma}+T_{a}^{\alpha \gamma}\right) \\
+v_{a}^{\gamma}\left(p_{a}^{\alpha \beta}+T_{a}^{\alpha \beta}\right)+Q_{a}^{\alpha \beta \gamma}+T_{a}^{\alpha \beta \gamma}+L_{a}^{\alpha \beta \gamma} .
\end{gathered}
$$

The pressure is the average of the square of the thermal velocity, when tensor $Q_{a}^{\alpha \beta \gamma}$ is the average of the product of three projections of the thermal velocity. Function $L_{a}^{\alpha \beta \gamma}$ presents quantum-thermal terms. For the BEC we have $p_{B}^{\alpha \beta}=0, Q_{B}^{\alpha \beta \gamma}=0, L_{B}^{\alpha \beta \gamma}=0$, since it has no contribution of the excited states. For symmetric equilibrium distributions we have $Q_{n}^{\alpha \beta \gamma}=0, L_{n}^{\alpha \beta \gamma}=0$. We generalize this conclusion for nonequilibrium states as the trivial equations of state for these functions. Tensor $T_{a}^{\alpha \beta \gamma}$ is

$$
T_{a}^{\alpha \beta \gamma}=-\frac{\hbar^{2}}{12 m^{2}} n_{a}\left(\partial^{\alpha} \partial^{\beta} v_{a}^{\gamma}+\partial^{\alpha} \partial^{\gamma} v_{a}^{\beta}+\partial^{\beta} \partial^{\gamma} v_{a}^{\alpha}\right) .
$$

This definition of tensor $T^{\alpha \beta \gamma}$ differs from equation (27) in Ref. 35] by extraction of the quantum Bohm potentials written together with pressure tensors in equation (21). Equation (27) in Ref. [35] contains approximate form of the quantum Bohm potential $T^{\alpha \beta}$. Equation (21) includes the quantum Bohm potential in its general form. Moreover, expression (22) is an exact formula obtained with no assumption about structure of the many-particle wave function like the first term in equation (23) in Ref. [35].

Equations (3)-20 are obtained in general form. The short-range nature of the inter-particle interaction is not used. Moreover, the traditional hydrodynamic equations are presented via the velocity field and the pressure tensor while equations (3)-(20) are written via the current and the momentum flux.

The method of the introduction of the velocity field in the equations of quantum hydrodynamics of spinless particles is presented in Refs. [2], [35]. The method of calculation of the terms containing interaction for the short-range interaction limit is also described in Refs. [2], 35]. Let us present results of application of these methods for finite temperature bosons. Moreover, we consider the short-range interaction in the first order by the interaction radius. 


\section{Appearance of the quantum fluctuations in the third rank tensor evolution equation}

Derivation of the quantum fluctuations requires the calculation of the time evolution of the current of the momentum flux $M^{\alpha \beta \gamma}$ (18). The method of derivation is similar to the equations obtained above. The time derivative of tensor $M^{\alpha \beta \gamma}$ acts on the wave function in its definition. The time derivative of the wave function is replaced by the Hamiltonian (1) in accordance with the many-particle microscopic Schrodinger equation $\imath \hbar \partial_{t} \Psi=\hat{H} \Psi$. It leads to the following expression:

$$
\begin{gathered}
\partial_{t} M^{\alpha \beta \gamma}=\frac{\imath}{\hbar} \int d R \sum_{i=1}^{N} \delta\left(\mathbf{r}-\mathbf{r}_{i}\right) \frac{1}{8 m_{i}^{3}}\left[\hat{H}^{*} \Psi^{*} \cdot \hat{p}_{i}^{\alpha} \hat{p}_{i}^{\beta} \hat{p}_{i}^{\gamma} \Psi\right. \\
-\Psi^{*} \hat{p}_{i}^{\alpha} \hat{p}_{i}^{\beta} \hat{p}_{i}^{\gamma} \hat{H} \Psi+\hat{p}_{i}^{\alpha *} \hat{H}^{*} \Psi^{*} \cdot \hat{p}_{i}^{\beta} \hat{p}_{i}^{\gamma} \Psi+\hat{p}_{i}^{\alpha *} \hat{p}_{i}^{\gamma *} \hat{H}^{*} \Psi^{*} \cdot \hat{p}_{i}^{\beta} \Psi \\
-\hat{p}_{i}^{\alpha *} \Psi^{*} \cdot \hat{p}_{i}^{\beta} \hat{p}_{i}^{\gamma} \hat{H} \Psi-\hat{p}_{i}^{\alpha *} \hat{p}_{i}^{\gamma *} \Psi^{*} \cdot \hat{p}_{i}^{\beta} \hat{H} \Psi
\end{gathered}
$$$$
\left.+\hat{p}_{i}^{\gamma *} \hat{H}^{*} \Psi^{*} \cdot \hat{p}_{i}^{\alpha} \hat{p}_{i}^{\beta} \Psi-\hat{p}_{i}^{\gamma *} \Psi^{*} \cdot \hat{p}_{i}^{\alpha} \hat{p}_{i}^{\beta} \hat{H} \Psi-\text { c.c. }\right]
$$

The part of the presented terms contains the Hamiltonian $\hat{H}$ under action of the momentum operators. We permute the Hamiltonian $\hat{H}$ and the operators acting on it. Hence, the result of permutation presented by terms where no operators act on the Hamiltonian $\hat{H}$. However, the terms containing the corresponding commutators appear. Therefore, all terms are combined in two groups:

$$
\begin{gathered}
\partial_{t} M^{\alpha \beta \gamma}=\frac{\imath}{\hbar} \int d R \sum_{i=1}^{N} \delta\left(\mathbf{r}-\mathbf{r}_{i}\right) \frac{1}{8 m_{i}^{3}}\left[\hat{H}^{*} \Psi^{*} \cdot \hat{p}_{i}^{\alpha} \hat{p}_{i}^{\beta} \hat{p}_{i}^{\gamma} \Psi\right. \\
-\Psi^{*} \hat{H} \hat{p}_{i}^{\alpha} \hat{p}_{i}^{\beta} \hat{p}_{i}^{\gamma} \Psi+\hat{H}^{*} \hat{p}_{i}^{\alpha *} \Psi^{*} \cdot \hat{p}_{i}^{\beta} \hat{p}_{i}^{\gamma} \Psi+\hat{H}^{*} \hat{p}_{i}^{\alpha *} \hat{p}_{i}^{\gamma *} \Psi^{*} \cdot \hat{p}_{i}^{\beta} \Psi \\
-\hat{p}_{i}^{\alpha *} \Psi^{*} \cdot \hat{H} \hat{p}_{i}^{\beta} \hat{p}_{i}^{\gamma} \Psi-\hat{p}_{i}^{\alpha *} \hat{p}_{i}^{\gamma *} \Psi^{*} \cdot \hat{H} \hat{p}_{i}^{\beta} \Psi \\
\left.+\hat{H}^{*} \hat{p}_{i}^{\gamma *} \Psi^{*} \cdot \hat{p}_{i}^{\alpha} \hat{p}_{i}^{\beta} \Psi-\hat{p}_{i}^{\gamma *} \Psi^{*} \cdot \hat{H}^{\hat{p}_{i}^{\alpha}} \hat{p}_{i}^{\beta} \Psi-c . c .\right] \\
+\frac{\imath}{\hbar} \int d R \sum_{i=1}^{N} \delta\left(\mathbf{r}-\mathbf{r}_{i}\right) \frac{1}{8 m_{i}^{3}}\left[-\Psi^{*}\left[\hat{p}_{i}^{\alpha} \hat{p}_{i}^{\beta} \hat{p}_{i}^{\gamma}, \hat{H}\right] \Psi\right. \\
+\left[\hat{p}_{i}^{\alpha *}, \hat{H}^{*}\right] \Psi^{*} \cdot \hat{p}_{i}^{\beta} \hat{p}_{i}^{\gamma} \Psi+\left[\hat{p}_{i}^{\alpha *} \hat{p}_{i}^{\gamma *}, \hat{H}^{*}\right] \Psi^{*} \cdot \hat{p}_{i}^{\beta} \Psi \\
-\hat{p}_{i}^{\alpha *} \Psi^{*} \cdot\left[\hat{p}_{i}^{\beta} \hat{p}_{i}^{\gamma}, \hat{H}\right] \Psi-\hat{p}_{i}^{\alpha *} \hat{p}_{i}^{\gamma *} \Psi^{*} \cdot\left[\hat{p}_{i}^{\beta}, \hat{H}\right] \Psi \\
\left.\left.\hat{H}^{*}\right] \Psi^{*} \cdot \hat{p}_{i}^{\alpha} \hat{p}_{i}^{\beta} \Psi-\hat{p}_{i}^{\gamma *} \Psi^{*} \cdot\left[\hat{p}_{i}^{\alpha} \hat{p}_{i}^{\beta}, \hat{H}^{\prime}\right] \Psi-c . c .\right] \cdot(24)
\end{gathered}
$$

The first group of terms leads to the divergence of the flux of tensor $M^{\alpha \beta \gamma}$. The second group of terms containing the commutators presents the interactions.

Final form of tensor $M^{\alpha \beta \gamma}$ evolution equation can be expressed in the following terms:

$$
\begin{gathered}
\partial_{t} M^{\alpha \beta \gamma}+\partial_{\delta} R^{\alpha \beta \gamma \delta}=\frac{\hbar^{2}}{4 m^{3}} n \partial_{\alpha} \partial_{\beta} \partial_{\gamma} V_{e x t} \\
-\frac{1}{m} \Pi^{\beta \gamma} \partial_{\alpha} V_{e x t}-\frac{1}{m} \Pi^{\alpha \gamma} \partial_{\beta} V_{e x t}-\frac{1}{m} \Pi^{\alpha \beta} \partial_{\gamma} V_{e x t} \\
+\frac{1}{m} F_{q f}^{\alpha \beta \gamma}+\frac{1}{m}\left(F^{\alpha \beta \gamma}+F^{\beta \gamma \alpha}+F^{\gamma \alpha \beta}\right),
\end{gathered}
$$

where

$$
F_{q f}^{\alpha \beta \gamma}=\frac{\hbar^{2}}{4 m^{2}} \int\left[\partial^{\alpha} \partial^{\beta} \partial^{\gamma} U\left(\mathbf{r}-\mathbf{r}^{\prime}\right)\right] n_{2}\left(\mathbf{r}, \mathbf{r}^{\prime}, t\right) d \mathbf{r}^{\prime}
$$

is the quantum force contribution leading to the quantum fluctuations, and

$$
F^{\alpha \beta \gamma}=-\int\left[\partial^{\alpha} U\left(\mathbf{r}-\mathbf{r}^{\prime}\right)\right] \Pi_{2}^{\beta \gamma}\left(\mathbf{r}, \mathbf{r}^{\prime}, t\right) d \mathbf{r}^{\prime}
$$

is the interaction contribution containing nonzero limit in the classical regime, with

$$
\begin{gathered}
\Pi_{2}^{\alpha \beta}\left(\mathbf{r}, \mathbf{r}^{\prime}, t\right)=\int d R \sum_{i, j \neq i} \frac{1}{4 m_{i}^{2}} \delta\left(\mathbf{r}-\mathbf{r}_{i}\right) \delta\left(\mathbf{r}^{\prime}-\mathbf{r}_{j}\right) \times \\
\times\left[\Psi^{*} \hat{p}_{i}^{\alpha} \hat{p}_{i}^{\beta} \Psi+\left(\hat{p}_{i}^{\beta}\right)^{*} \Psi^{*} \hat{p}_{i}^{\alpha} \Psi+\text { c.c. }\right] .
\end{gathered}
$$

Tensor $\Pi_{2}^{\alpha \beta}\left(\mathbf{r}, \mathbf{r}^{\prime}, t\right)$ can be simplified in the correlationless regime to the following form $\Pi_{2}^{\alpha \beta}\left(\mathbf{r}, \mathbf{r}^{\prime}, t\right)=\Pi^{\alpha \beta}(\mathbf{r}, t)$. $n\left(\mathbf{r}^{\prime}, t\right)$. However, the correlations caused by the symmetrization of the bosonic many-particle wave function are used below.

Terms $F^{\alpha \beta \gamma}$ and $F_{q f}^{\alpha \beta \gamma}$ are the third rank force tensors describing the interparticle interaction. However, equation (25) contains the flux of tensor $M^{\alpha \beta \gamma}$ which is the fourth rank tensor appearing in the following form:

$$
\begin{gathered}
R^{\alpha \beta \gamma \delta}=\int d R \sum_{i=1}^{N} \delta\left(\mathbf{r}-\mathbf{r}_{i}\right) \frac{1}{16 m_{i}^{4}}\left[\Psi^{*} \hat{p}_{i}^{\alpha} \hat{p}_{i}^{\beta} \hat{p}_{i}^{\gamma} \hat{p}_{i}^{\delta} \Psi\right. \\
+\hat{p}_{i}^{\alpha *} \Psi^{*} \hat{p}_{i}^{\beta} \hat{p}_{i}^{\gamma} \hat{p}_{i}^{\delta} \Psi+\hat{p}_{i}^{\beta *} \Psi^{*} \hat{p}_{i}^{\alpha} \hat{p}_{i}^{\gamma} \hat{p}_{i}^{\delta} \Psi+\hat{p}_{i}^{\gamma *} \Psi^{*} \hat{p}_{i}^{\alpha} \hat{p}_{i}^{\beta} \hat{p}_{i}^{\gamma} \Psi \\
+\hat{p}_{i}^{\delta *} \Psi^{*} \hat{p}_{i}^{\alpha} \hat{p}_{i}^{\beta} \hat{p}_{i}^{\gamma} \Psi+\hat{p}_{i}^{\alpha *} \hat{p}_{i}^{\delta *} \Psi^{*} \hat{p}_{i}^{\beta} \hat{p}_{i}^{\gamma} \Psi \\
\left.+\hat{p}_{i}^{\alpha *} \hat{p}_{i}^{\gamma *} \Psi^{*} \hat{p}_{i}^{\beta} \hat{p}_{i}^{\delta} \Psi+\hat{p}_{i}^{\gamma *} \hat{p}_{i}^{\delta *} \Psi^{*} \hat{p}_{i}^{\alpha} \hat{p}_{i}^{\beta} \Psi+\text { c.c. }\right] .
\end{gathered}
$$

Equation (25) is obtained for bosons with the arbitrary temperature. It can be separated on two equations for 
two following subsystems: the BEC and the normal fluid. All terms in equation (25) are additive on the particles. Therefore, they are additive on the subsystems. Hence, the structure of the partial equations is identical to the structure of equation (25):

$$
\begin{gathered}
\partial_{t} M_{a}^{\alpha \beta \gamma}+\partial_{\delta} R_{a}^{\alpha \beta \gamma \delta}=-\frac{1}{m} n_{a} \partial_{\alpha} \partial_{\beta} \partial_{\gamma} V_{e x t} \\
-\frac{1}{m} \Pi_{a}^{\beta \gamma} \partial_{\alpha} V_{e x t}-\frac{1}{m} \Pi_{a}^{\alpha \gamma} \partial_{\beta} V_{e x t}-\frac{1}{m} \Pi_{a}^{\alpha \beta} \partial_{\gamma} V_{e x t} \\
+\frac{1}{m} F_{a, q f}^{\alpha \beta \gamma}+\frac{1}{m}\left(F_{a}^{\alpha \beta \gamma}+F_{a}^{\beta \gamma \alpha}+F_{a}^{\gamma \alpha \beta}\right),
\end{gathered}
$$

where subindex $a$ equal $B$ for the BEC and $n$ for the normal fluid.

The fourth rank kinematic tensor $R_{a}^{\alpha \beta \gamma \delta}$ (29) has the following form after the introduction of the velocity field via the Madelung transformation of the many-particle wave function:

$$
\begin{gathered}
R_{a}^{\alpha \beta \gamma \delta}=n_{a} v_{a}^{\alpha} v_{a}^{\beta} v_{a}^{\gamma} v_{a}^{\delta} \\
+v_{a}^{\alpha} v_{a}^{\delta}\left(p_{a}^{\beta \gamma}+T_{a}^{\beta \gamma}\right)+v_{a}^{\beta} v_{a}^{\delta}\left(p_{a}^{\alpha \gamma}+T_{a}^{\alpha \gamma}\right)+v_{a}^{\gamma} v_{a}^{\delta}\left(p_{a}^{\alpha \beta}+T_{a}^{\alpha \beta}\right) \\
+v_{a}^{\alpha} v_{a}^{\gamma}\left(p_{a}^{\beta \delta}+T_{a}^{\beta \delta}\right)+v_{a}^{\beta} v_{a}^{\gamma}\left(p_{a}^{\alpha \delta}+T_{a}^{\alpha \delta}\right)+v_{a}^{\alpha} v_{a}^{\beta}\left(p_{a}^{\gamma \delta}+T_{a}^{\gamma \delta}\right) \\
+v_{a}^{\alpha} Q_{a}^{\beta \gamma \delta}+v_{a}^{\beta} Q_{a}^{\alpha \gamma \delta}+v_{a}^{\gamma} Q_{a}^{\alpha \beta \delta}+v_{a}^{\delta} Q_{a}^{\alpha \beta \gamma} \\
+Q_{a}^{\alpha \beta \gamma \delta}+T_{a}^{\alpha \beta \gamma \delta}+L_{a}^{\alpha \beta \gamma \delta} .
\end{gathered}
$$

This structure shows some similarity to the representations for the second rank tensor momentum flux (11) and for the third rank tensor (21), where the higher rank tensors are partially transformed via the concentration, velocity field and, if possible, via tensors of smaller rank. However, this transformation is partial since there is the tensor of the equal rank, but defined in the comoving frame. Moreover, this final tensor is splitted on few parts. It is two parts for the second rank tensor momentum flux, where we have the kinetic pressure (quasi-classical part of thermal nature) and the quantum Bohm potential (the quantum part). There are three parts for the third rank tensor $M^{\alpha \beta \gamma}$. They are the quasi-classical part of thermal nature, the quantum part, and the combined thermal-quantum part. For the fourth rank tensor we also have three parts: the quasi-classical part of thermal nature $Q_{a}^{\alpha \beta \gamma \delta}$, the quantum part $T_{a}^{\alpha \beta \gamma \delta}$, and the combined thermal-quantum part $L_{a}^{\alpha \beta \gamma \delta}$.

Developed model shows that arbitrary quantum system can be modeled via the hydrodynamic equations which are traditionally associated with the fluid dynamics. Quantum systems demonstrates that each particle shows the properties of the wave and this wave-like behavior is incorporated in the quantum hydrodynamic model. This conclusion follows from the fact that the quantum hydrodynamic is derived from the Schrodinger equations which contains these information. These general concept is illustrated for the ultracold bosons, but the quantum hydrodynamic method can be applied to other physical systems. This similarity between quantum behavior and the dynamics of fluids recently found unusual realization. It is experimentally found that classic fluid objects demonstrate the quantum-like behavior [36], 37], [38]. It is observed as the millimetric droplet walking on the surface of vibrating fluids, where the motion of droplets is affected by the resonant interaction with their own wave field [37], 38]. Systems walking droplets demonstrate various quantum effects [39], [40], [41].

\section{CONTRIBUTION OF INTERACTION IN THE QUANTUM HYDRODYNAMIC EQUATIONS}

Equations (6), (16), and (25) contain terms describing interaction. Approximate forms of these force fields of different tensor ranks are necessary to get a truncated set of equations. In our case, it is necessary to include the short-range nature of the potential of the interparticle interaction. Moreover, the weak interaction limit is considered. These two assumptions are used to get simplified form of $F^{\alpha}, F^{\alpha \beta}, F^{\alpha \beta \gamma}$ and $F_{q f}^{\alpha \beta \gamma}$ in this section.

\section{A. Interaction terms in the Euler equation}

The short-range interaction in the Euler for the single species of quantum particles can be written as the divergence of the symmetric quantum stress tensor $F^{\alpha}=$ $-\partial^{\beta} \sigma^{\alpha \beta}$.

The first order by the interaction radius approximation gives the following expression for the quantum stress tensor (see also [2])

$$
\begin{gathered}
\sigma^{\alpha \beta}(\mathbf{r}, t)=-\frac{1}{2} \int d R \sum_{i, j . i \neq j} \delta\left(\mathbf{r}-\mathbf{R}_{i j}\right) \times \\
\times \frac{r_{i j}^{\alpha} r_{i j}^{\beta}}{\left|\mathbf{r}_{i j}\right|} \frac{\partial U\left(\mathbf{r}_{i j}\right)}{\partial\left|\mathbf{r}_{i j}\right|} \Psi^{*}\left(R^{\prime}, t\right) \Psi\left(R^{\prime}, t\right),
\end{gathered}
$$

where $R^{\prime}=\left\{\ldots, \mathbf{R}_{i j}, \ldots, \mathbf{R}_{i j}, \ldots\right\}$ with vector $\mathbf{R}_{i j}$ located at $i$-th and $j$-th places.

Expression (32) can be rewritten in terms of twoparticle concentration

$$
\sigma^{\alpha \beta}(\mathbf{r}, t)=-\frac{1}{2} \operatorname{Tr}\left(n_{2}\left(\mathbf{r}, \mathbf{r}^{\prime}, t\right)\right) \int d \mathbf{r} \frac{r^{\alpha} r^{\beta}}{r} \frac{\partial U(r)}{\partial r},
$$

where the notion of trace is used

$$
\operatorname{Tr} f\left(\mathbf{r}, \mathbf{r}^{\prime}\right)=f(\mathbf{r}, \mathbf{r}) .
$$


Consideration of the short-range interaction leads to the separation of integral containing the potential of interaction. So, the characteristic of interaction does not depend on the motion or position of particles. This integral simplifies in the following way

$$
\int \frac{r^{\alpha} r^{\beta}}{r} \frac{\partial U}{\partial r} d \mathbf{r}=\frac{1}{3} \delta^{\alpha \beta} \int r U^{\prime} d \mathbf{r}=-\delta^{\alpha \beta} \int U d \mathbf{r}
$$

The last integral in this expression is denoted as $g=$ $\int U d \mathbf{r}$.

The two-particle concentration can be calculated in the weakly interacting limit (see [2])

$$
n_{2}\left(\mathbf{r}, \mathbf{r}^{\prime}, t\right)=n(\mathbf{r}, t) n\left(\mathbf{r}^{\prime}, t\right)+\left|\rho\left(\mathbf{r}, \mathbf{r}^{\prime}, t\right)\right|^{2}+\wp\left(\mathbf{r}, \mathbf{r}^{\prime}, t\right),
$$

where

$$
n(\mathbf{r}, t)=\sum_{f} n_{f} \varphi_{f}^{*}(\mathbf{r}, t) \varphi_{f}(\mathbf{r}, t)
$$

is the expression of concentration (2) in terms of the single particle wave functions $\varphi_{f}(\mathbf{r}, t)$,

$$
\rho\left(\mathbf{r}^{\prime}, \mathbf{r}, t\right)=\sum_{f} n_{f} \varphi_{f}^{*}(\mathbf{r}, t) \varphi_{f}\left(\mathbf{r}^{\prime}, t\right)
$$

is the density matrix, and

$$
\wp\left(\mathbf{r}, \mathbf{r}^{\prime}, t\right)=\sum_{f} n_{f}\left(n_{f}-1\right)\left|\varphi_{f}(\mathbf{r}, t)\right|^{2}\left|\varphi_{f}\left(\mathbf{r}^{\prime}, t\right)\right|^{2},
$$

The last term in equation describes interaction of pairs of particles being in the same quantum state. It can be seen from the existence of single quantum number $g$ in all wave are single-particle wave functions.

Expression (36) can be substituted in the general expression of the force field (8). However, equation (8) does not contain information about the short-range nature of considered interaction. The first and second terms are related to particles located in different quantum states. It cannot be seen from equation (36), but it follows from intermediate terms which can be found in Ref. [2].

The trace of the two-particle concentration entering the quantum stress tensor has the following form

$$
\operatorname{Trn}_{2}\left(\mathbf{r}, \mathbf{r}^{\prime}, t\right) \approx 2\left(n^{2}\right)^{\prime}+n_{B}^{2},
$$

where the first term on the right-hand side symbol' means that the product of concentrations is related to the particles in different quantum states. Therefore, the first term has no $n_{B}^{2}$ contribution from selfaction of BEC. The dropped terms are described in Ref. [42].

Present explicit contribution of the BEC concentration $n_{B}$ and the concentration of normal fluid $n_{n}$ in the first term on the right-hand side of equation (40):

$$
\left(n^{2}\right)^{\prime}=\left(\left(n_{B}+n_{n}\right)\left(n_{B}+n_{n}\right)\right)^{\prime}=\left(n_{n}^{2}+2 n_{B} n_{n}\right) .
$$

The last term in equation (40) appears for particles being in BEC. While the first term on the right-hand side in equation (40) related to interaction of particles being in different quantum states. Hence, it gives contribution for the interaction between BEC and normal fluid and for the interaction between bosons belonging to normal fluid.

Full expression of the quantum stress tensor for the bosons at finite temperature can be written in terms of the concentration of $\mathrm{BEC}$ and the concentration of normal fluid:

$$
\sigma^{\alpha \beta}=\frac{1}{2} g \delta^{\alpha \beta}\left(2 n_{n}^{2}+4 n_{B} n_{n}+n_{B}^{2}\right) .
$$

If we consider dynamics of BEC or normal fluid we cannot use the notion of the quantum stress tensor $\sigma^{\alpha \beta}$ for the interaction of subspecies as it is for the interaction of different species.

The first (last) term in equation (42) contains the selfaction of the normal fluid (of the BEC). The second term in equation (42) presents the interaction between the BEC and normal fluid.

If we consider dynamics of BEC we need to extract force caused by the BEC and normal fluid acting on the BEC. This force is the superposition of a part of the second term in equation (42) and the last term in equation (42):

$$
F_{B}^{\alpha}=-g n_{B} \partial^{\alpha}\left(2 n_{n}+n_{B}\right) .
$$

The second term in equation (42) can be rewritten as follows $F_{2}^{\alpha}=-2 g\left(n_{B} \partial^{\alpha} n_{n}+n_{n} \partial^{\alpha} n_{B}\right)$. The first part of this expression is used in equation (43).

If we consider dynamics of normal fluid it means that the source of field in the first term of $n_{2}$ can be the normal fluid and the BEC, hence the last term gives no contribution in this case in equation (42):

$$
F_{n}^{\alpha}=-2 g n_{n} \partial^{\alpha}\left(n_{n}+n_{B}\right) .
$$

The nonsymmetric decomposition allows to use the notion of the NLSE. It is necessary condition to have the GP equation at finite temperatures. Moreover, the nonsymmetric form is traditionally used in literature [32]. Same chose is made at analysis $j_{2}^{\beta}$ below.

\section{Nonlinear Schrodinger equations}

Dropping the pressure of normal fluid and using the quantum Bohm potential in form (12) we find a closed set of hydrodynamic equations. Introducing the macroscopic wave function for both the BEC and the normal fluid for the potential velocity fields as $\Phi_{a}=\sqrt{n_{a}} e^{\imath m \phi_{a} / \hbar}$, where $\phi_{a}$ is the potential of the velocity field $\mathbf{v}_{a}=-\nabla \phi_{a}$.

$$
\imath \hbar \partial_{t} \Phi_{B}=\left(-\frac{\hbar^{2} \nabla^{2}}{2 m}+V_{e x t}+g\left(n_{B}+2 n_{n}\right)\right) \Phi_{B},
$$

and

$$
\imath \hbar \partial_{t} \Phi_{n}=\left(-\frac{\hbar^{2} \nabla^{2}}{2 m}+V_{e x t}+2 g\left(n_{B}+n_{n}\right)\right) \Phi_{n} .
$$


The kinetic energy (the first term on the right-hand side of equations (45) and (46) ) corresponds to the application of the noninteracting limit for the quantum Bohm potential for the BEC and for the normal fluid. (46).

The pressure of the normal fluid is dropped in equation

Equations (45), (46) correspond to equations 127-129 given in Ref. [1] while there is a difference in the form of presentation.

Therefore, the account of the pressure evolution together with the pressure flux evolution gives the generalization of the model presented with the nonlinear Schrodinger equations (45), (46). Necessity of additional equations is demonstrated in Refs. [4], [43], [44] if one wants to include the quantum fluctuations.

\section{B. Interaction terms in the pressure evolution equation}

General form of the pressure evolution equation contains the interaction via the force second rank tensor field. Its main contribution is obtained in the first order by the interaction radius. The result appears in the following form

$$
\begin{gathered}
F^{\alpha \beta}(\mathbf{r}, t)=\frac{1}{8 m^{2}} \partial^{\gamma} \int d R \sum_{i, j ; i \neq j} \delta\left(\mathbf{r}-\mathbf{R}_{i j}\right) \times \\
\times \frac{r_{i j}^{\beta} r_{i j}^{\gamma}}{\left|\mathbf{r}_{i j}\right|} \frac{\partial U\left(\mathbf{r}_{i j}\right)}{\partial\left|\mathbf{r}_{i j}\right|}\left[\Psi^{*}\left(R^{\prime}, t\right)\left(\hat{p}_{(1)}^{\alpha}+\hat{p}_{(2)}^{\alpha}\right) \Psi\left(R^{\prime}, t\right)+c . c .\right] \\
-\frac{1}{8 m^{2}} \int d R \sum_{i, j ; i \neq j} \delta\left(\mathbf{r}-\mathbf{R}_{i j}\right) \frac{r_{i j}^{\alpha} r_{i j}^{\gamma}}{\left|\mathbf{r}_{i j}\right|} \frac{\partial U\left(\mathbf{r}_{i j}\right)}{\partial\left|\mathbf{r}_{i j}\right|} \times \\
\times\left[\left(\partial_{(1)}^{\gamma}-\partial_{(2)}^{\gamma}\right) \Psi^{*}\left(R^{\prime}, t\right)\left(\hat{p}_{(1)}^{\alpha}-\hat{p}_{(2)}^{\alpha}\right) \Psi\left(R^{\prime}, t\right)\right. \\
\left.+\Psi^{*}\left(R^{\prime}, t\right)\left(\partial_{(1)}^{\gamma}-\partial_{(2)}^{\gamma}\right)\left(\hat{p}_{(1)}^{\alpha}-\hat{p}_{(2)}^{\alpha}\right) \Psi\left(R^{\prime}, t\right)+c . c .\right] .
\end{gathered}
$$

Form (47) appears at the expansion of the force tensor field (17) using the short-range nature of interaction (see [2] for the method described for the force field, or [35] for application of this method to fermions).

For the force tensor field $F^{\alpha \beta}$ we can present the intermediate expressions like equations (33) and (36) obtained for the force field $F^{\alpha}=-\partial^{\beta} \sigma^{\alpha \beta}$. However, similar expressions obtained for $F^{\alpha \beta}$ are rather large. Hence, we start the presentation with equation similar to equation (40) obtained after taking trace of the intermediate expressions.

Therefore, we obtain the following simplification of equation (47) for the force tensor field $F^{\alpha \beta}$ :

$$
F^{\alpha \beta}=-\frac{g}{4 m^{2}} \partial^{\beta}\left[2\left(n \Lambda^{\alpha}\right)^{\prime}+n_{B} \Lambda_{B}^{\alpha}\right]
$$

$$
+\frac{\imath}{\hbar} \frac{g}{4 m^{2}}\left[2\left(n r^{\alpha \beta}\right)^{\prime}-2\left(\Lambda^{\alpha} \Lambda^{\beta}\right)^{\prime}+n_{B} r_{B}^{\alpha \beta}-\Lambda_{B}^{\alpha} \Lambda_{B}^{\beta}\right]+c . c .,
$$

where we use the intermediate functions $\Lambda^{\alpha}$ and $r^{\alpha \beta}$ with the following definitions:

$$
\Lambda^{\alpha}=\sum_{f} n_{f} \varphi_{f}^{*} \hat{p}^{\alpha} \varphi_{f}=m j^{\alpha}-\imath \frac{\hbar}{2} \partial^{\alpha} n
$$

and

$$
\begin{gathered}
r^{\alpha \beta}=\sum_{f} n_{f} \varphi_{f}^{*} \hat{p}^{\alpha} \hat{p}^{\beta} \varphi_{f} \\
=m^{2}\left(n v^{\alpha} v^{\beta}+p^{\alpha \beta}-\frac{\hbar^{2}}{m^{2}} \sum_{f} n_{f} a_{f} \partial^{\alpha} \partial^{\beta} a_{f}\right) \\
-\imath \frac{m \hbar}{2}\left[\partial^{\alpha}\left(n v^{\beta}\right)+\partial^{\beta}\left(n v^{\alpha}\right)\right] .
\end{gathered}
$$

The calculation of functions $\Lambda^{\alpha}$ and $r^{\alpha \beta}$ includes the Madelung transformation of the single-particle wave functions $\varphi_{f}(r, t)=\sqrt{a_{f}} e^{\imath S_{f}}$. Next, we use the following definitions of the velocity field and the pressure tensor in terms of the single-particle wave functions $n v^{\alpha}=\sum_{f} n_{f} a_{f}^{2}\left(\hbar \partial^{\alpha} S_{f} / m\right)$, and $p^{\alpha \beta}=\sum_{f} n_{f} a_{f}^{2} u_{f}^{\alpha} u_{f}^{\beta}$, where $u_{f}^{\alpha}=\left(\hbar \partial^{\alpha} S_{f} / m\right)-v^{\alpha}$.

Let us represent terms like $\left(n \Lambda^{\alpha}\right)^{\prime}$ in the explicit form:

$$
\begin{gathered}
F^{\alpha \beta}=-\frac{g}{4 m^{2}} \partial^{\beta}\left[2 n_{n} \Lambda_{n}^{\alpha}\right. \\
\left.+2 n_{n} \Lambda_{B}^{\alpha}+2 n_{B} \Lambda_{n}^{\alpha}+n_{B} \Lambda_{B}^{\alpha}\right] \\
+\frac{\imath}{\hbar} \frac{g}{4 m^{2}}\left[2 n_{n} r_{n}^{\alpha \beta}-2 \Lambda_{n}^{\alpha} \Lambda_{n}^{\beta}+2 n_{n} r_{B}^{\alpha \beta}-2 \Lambda_{n}^{\alpha} \Lambda_{B}^{\beta}\right. \\
\left.2 n_{B} r_{n}^{\alpha \beta}-2 \Lambda_{B}^{\alpha} \Lambda_{n}^{\beta}+n_{B} r_{B}^{\alpha \beta}-\Lambda_{B}^{\alpha} \Lambda_{B}^{\beta}\right]+c . c . .
\end{gathered}
$$

Further calculation gives the representation of tensor $F^{\alpha \beta}$ in term of hydrodynamic functions:

$$
\begin{gathered}
F^{\alpha \beta}=-\frac{g}{2 m} \partial^{\beta}\left[2 n_{n} j_{n}^{\alpha}+2 n_{n} j_{B}^{\alpha}+2 n_{B} j_{n}^{\alpha}+n_{B} j_{B}^{\alpha}\right] \\
+\frac{g}{4 m}\left[2 n_{n}\left(\partial j_{n}+\partial j_{n}\right)-2 j_{n} \partial n_{n}-2 j_{n} \partial n_{n}\right. \\
+2 n_{n}\left(\partial^{\alpha} j_{B}^{\beta}+\partial^{\beta} j_{B}^{\alpha}\right)-2 j_{B}^{\alpha} \partial^{\beta} n_{n}-2 j_{B}^{\beta} \partial^{\alpha} n_{n} \\
+2 n_{B}\left(\partial^{\alpha} j_{n}^{\beta}+\partial^{\beta} j_{n}^{\alpha}\right)-2 j_{n}^{\alpha} \partial^{\beta} n_{B}-2 j_{n}^{\beta} \partial^{\alpha} n_{B} \\
\left.+n_{B}\left(\partial^{\alpha} j_{B}^{\beta}+\partial^{\beta} j_{B}^{\alpha}\right)-j_{B}^{\alpha} \partial^{\beta} n_{B}-j_{B}^{\beta} \partial^{\alpha} n_{B}\right] .
\end{gathered}
$$


The momentum flux evolution equation contains the symmetric combination of the force tensor fields $F^{\alpha \beta}$ :

$$
\begin{gathered}
F^{\alpha \beta}+F^{\beta \alpha}=-\frac{g}{m}\left[2\left(j_{n}^{\alpha} \partial^{\beta} n_{n}+j_{n}^{\beta} \partial^{\alpha} n_{n}\right)\right. \\
+2\left(j_{n}^{\alpha} \partial^{\beta} n_{B}+j_{n}^{\beta} \partial^{\alpha} n_{B}\right) \\
\left.+2\left(j_{B}^{\alpha} \partial^{\beta} n_{n}+j_{B}^{\beta} \partial^{\alpha} n_{n}\right)+j_{B}^{\alpha} \partial^{\beta} n_{B}+j_{B}^{\beta} \partial^{\alpha} n_{B}\right] .
\end{gathered}
$$

The zero temperature analysis demonstrates that there is nonzero pressure for the BECs, caused by the quantum fluctuations entering the set of hydrodynamic equations via the evolution of the pressure flux [4], 43], [44]. The pressure also exists for the normal fluid. So, we make decomposition of the momentum flux evolution equation on two partial equations for $\Pi_{n}^{\alpha \beta}$ and $\Pi_{B}^{\alpha \beta}$. Formally this decomposition is presented with equation (20). To complete this procedure we need to split the force tensor field $F^{\alpha \beta}+F^{\beta \alpha}=F_{B}^{\alpha \beta}+F_{B}^{\beta \alpha}+F_{n}^{\alpha \beta}+F_{n}^{\beta \alpha}$, where

$$
\begin{gathered}
F_{B}^{\alpha \beta}+F_{B}^{\beta \alpha}=-\frac{g}{m}\left[2\left(j_{B}^{\alpha} \partial^{\beta} n_{n}+j_{B}^{\beta} \partial^{\alpha} n_{n}\right)\right. \\
\left.+j_{B}^{\alpha} \partial^{\beta} n_{B}+j_{B}^{\beta} \partial^{\alpha} n_{B}\right]
\end{gathered}
$$

and

$$
F_{n}^{\alpha \beta}+F_{n}^{\beta \alpha}=-\frac{g}{m}\left[2\left(j_{n}^{\alpha} \partial^{\beta} n_{n}+j_{n}^{\beta} \partial^{\alpha} n_{n}\right)\right.
$$

$$
\left.+2\left(j_{n}^{\alpha} \partial^{\beta} n_{B}+j_{n}^{\beta} \partial^{\alpha} n_{B}\right)\right] .
$$

After extraction of the pressure tensor $p^{\alpha \beta}$ from the momentum flux evolution $\Pi^{\alpha \beta}$ we have extra contribution of the interaction in the pressure evolution equation in compare with equations (20). It contains the following contribution $F^{\alpha \beta}-v^{\beta} F^{\alpha}$.

Using equations (43), (44), (55), (54) find $F^{\alpha \beta}+F^{\alpha \beta}-$ $v^{\alpha} F^{\beta}-v^{\beta} F^{\alpha}=0$ for the BECs and for the normal fluid.

A pressure evolution equation is used in [45] for bosons above the critical temperature. Equation 4 of Ref. 45] contains the force in the following form $n \mathbf{v} \cdot \mathbf{F}$ which generally differs from $F^{\alpha \beta}-v^{\beta} F^{\alpha}$ obtained above.

\section{The short-range interaction in the third rank tensor evolution equation}

The third rank tensor $M^{\alpha \beta \gamma}$ (25) evolution equation contains two kinds of the third rank force tensors $F^{\alpha \beta \gamma}$ (27) and $F_{q f}^{\alpha \beta \gamma}(26)$. Consider them separately.

1. Quasi-classical third rank force tensor

Tensor $F_{q f}^{\alpha \beta \gamma}(26)$ is proportional to the Planck constant, so it goes to zero in the classical limit. The third rank force tensor $F^{\alpha \beta \gamma}$ (27) is different, it has nonzero limit in the quasiclassical regime. However, we are interested in the value of tensor $F^{\alpha \beta \gamma}$ (27) in one of quantum regimes for the degenerate bosons.

We calculate the third rank force tensor $F^{\alpha \beta \gamma}(27)$ in the first order by the interaction radius appears in the following form

$$
\begin{aligned}
& F^{\alpha \beta \gamma}(\mathbf{r}, t)=\frac{1}{8 m^{3}} \partial^{\mu} \int d R \sum_{i, j ; i \neq j} \delta\left(\mathbf{r}-\mathbf{R}_{i j}\right) \frac{r_{i j}^{\mu} r_{i j}^{\gamma}}{\left|\mathbf{r}_{i j}\right|} \frac{\partial U\left(\mathbf{r}_{i j}\right)}{\partial\left|\mathbf{r}_{i j}\right|}\left[\Psi^{*}\left(R^{\prime}, t\right) \hat{p}_{(1)}^{\alpha} \hat{p}_{(1)}^{\beta} \Psi\left(R^{\prime}, t\right)+\hat{p}_{(1)}^{\beta *} \Psi^{*}\left(R^{\prime}, t\right) \hat{p}_{(1)}^{\alpha} \Psi\left(R^{\prime}, t\right)+c . c .\right] \\
& -\frac{1}{8 m^{3}} \int d R \sum_{i, j ; i \neq j} \delta\left(\mathbf{r}-\mathbf{R}_{i j}\right) \frac{r_{i j}^{\mu} r_{i j}^{\gamma}}{\left|\mathbf{r}_{i j}\right|} \frac{\partial U\left(\mathbf{r}_{i j}\right)}{\partial\left|\mathbf{r}_{i j}\right|}\left[\left(\partial_{(1)}^{\mu}-\partial_{(2)}^{\mu}\right) \Psi^{*}\left(R^{\prime}, t\right) \hat{p}_{(1)}^{\alpha} \hat{p}_{(1)}^{\beta} \Psi\left(R^{\prime}, t\right)\right. \\
& \left.+\Psi^{*}\left(R^{\prime}, t\right)\left(\partial_{(1)}^{\mu}-\partial_{(2)}^{\mu}\right) \hat{p}_{(1)}^{\alpha} \hat{p}_{(1)}^{\beta} \Psi\left(R^{\prime}, t\right)+\hat{p}_{(1)}^{\beta *}\left(\partial_{(1)}^{\mu}-\partial_{(2)}^{\mu}\right) \Psi^{*}\left(R^{\prime}, t\right) \hat{p}_{(1)}^{\alpha} \Psi\left(R^{\prime}, t\right)+\hat{p}_{(1)}^{\beta *} \Psi^{*}\left(R^{\prime}, t\right)\left(\partial_{(1)}^{\mu}-\partial_{(2)}^{\mu}\right) \hat{p}_{(1)}^{\alpha} \Psi\left(R^{\prime}, t\right)+c . c .\right]
\end{aligned}
$$

Here, the part of expression for $F^{\alpha \beta \gamma}$ containing the interaction potential appears as the independent multiplier. It has same form as the integral in the Euler equation (35). Hence, tensor $F^{\alpha \beta \gamma}$ is proportional to the Groos-Pitaevskii interaction constant.

Further calculation in the weakly interacting limit, following the method described in Ref. 2], gives an intermediate representation of the third rank force tensor:

$$
F^{\alpha \beta \gamma}(\mathbf{r}, t)=-\frac{g}{4 m^{3}}\left[\Pi_{B}^{\alpha \beta} \partial^{\gamma} n_{B}+\Pi^{\alpha \beta} \partial^{\gamma} n+\left(n \sum_{f} n_{f} \partial^{\gamma} \varphi_{f}^{*} \hat{p}^{\alpha} \hat{p}^{\beta} \varphi_{f}+\frac{\imath}{\hbar} \Lambda^{\gamma} r^{\alpha \beta}-\frac{\imath}{\hbar} \Lambda^{\alpha *} \kappa^{\gamma \beta}+\frac{\imath}{\hbar} \Lambda^{\beta} \kappa^{\alpha \gamma}+c . c .\right)\right]
$$


where

$$
\kappa^{\alpha \beta}=\sum_{f} n_{f} p^{\alpha} \varphi_{f}^{*} \cdot \hat{p}^{\beta} \varphi_{f}
$$

Function $\kappa^{\alpha \beta}$ is the nonsymmetric tensor. It has symmetric real part and the antisymmetric imaginary part:

$$
\begin{gathered}
\kappa^{\alpha \beta}=m^{2}\left(n v^{\alpha} v^{\beta}+p^{\alpha \beta}+\frac{\hbar^{2}}{m^{2}} \sum_{f} n_{f} \partial^{\alpha} \cdot a_{f} \partial^{\beta} a_{f}\right) \\
-\frac{1}{2} \imath m \hbar\left[v^{\alpha} \partial^{\beta} n-v^{\beta} \partial^{\alpha} n+\sum_{f} n_{f} a_{f}\left(u^{\alpha} \partial^{\beta} a_{f}-u^{\beta} \partial^{\alpha} a_{f}\right)\right] .
\end{gathered}
$$

No specific notation is introduced for the third rank tensor $\sum_{f} n_{f} \partial^{\gamma} \varphi_{f}^{*} \hat{p}^{\alpha} \hat{p}^{\beta} \varphi_{f}$. In our calculations we need its imaginary part multiplied by 2 :

$$
\begin{gathered}
\sum_{f} n_{f} \partial^{\gamma} \varphi_{f}^{*} \hat{p}^{\alpha} \hat{p}^{\beta} \varphi_{f}+c . c .=2 m^{2}\left[\frac{1}{2} \partial^{\gamma} n \cdot v^{\alpha} v^{\beta}-\partial^{\alpha} n \cdot v^{\beta} v^{\gamma}-\partial^{\beta} n \cdot v^{\alpha} v^{\gamma}-n v^{\gamma}\left(\partial^{\beta} v^{\alpha}+\partial^{\alpha} v^{\beta}\right)\right. \\
+\sum_{f} n_{f} a_{f}\left(\partial^{\gamma} a_{f}\right) u_{f}^{\alpha} u_{f}^{\beta}-\frac{1}{2} \partial^{\beta} p^{\alpha \gamma}-\frac{1}{2} \partial^{\alpha} p^{\beta \gamma}+\frac{1}{2} \sum_{f} n_{f} a_{f}^{2}\left(u_{f}^{\beta} \partial^{\alpha} u_{f}^{\gamma}+u_{f}^{\alpha} \partial^{\beta} u_{f}^{\gamma}\right) \\
\left.\left.+v^{\alpha} \sum_{f} n_{f} a_{f}\left(\partial^{\gamma} a_{f} \cdot u_{f}^{\beta}-\partial^{\beta} a_{f} \cdot u_{f}^{\gamma}\right)+v^{\beta} \sum_{f} n_{f} a_{f}\left(\partial^{\gamma} a_{f} \cdot u_{f}^{\alpha}-\partial^{\alpha} a_{f} \cdot u_{f}^{\gamma}\right)-\frac{\hbar^{2}}{m^{2}} \sum_{f} n_{f} \partial^{\gamma} a_{f} \cdot \partial^{\alpha} \partial^{\beta} a_{f}\right)\right] .
\end{gathered}
$$

Equation (57) includes term $\Pi_{B}^{\alpha \beta} \partial^{\gamma} n_{B}$ which describes full contribution of the BEC in $F^{\alpha \beta \gamma}$.

The second term in equation (57) is an analog of the first term in equation (36). All following terms in equation (57) are the analog of the second term in equation (36). It can be interpreted as the exchange interaction.

Further calculation of the (57) gives the partially truncated expression mainly presented via the macroscopic hydrodynamic functions:

$$
\begin{aligned}
& F^{\alpha \beta \gamma}(\mathbf{r}, t)=- \frac{g}{4}\left[4 \Pi_{B}^{\alpha \beta} \partial^{\gamma} n_{B}+4 \Pi^{\alpha \beta} \partial^{\gamma} n+\partial^{\alpha} n\left(\Pi^{\beta \gamma}+\frac{\hbar^{2}}{4 m^{2}} \partial^{\beta} \partial^{\gamma} n\right)+\partial^{\beta} n\left(\Pi^{\alpha \gamma}+\frac{\hbar^{2}}{4 m^{2}} \partial^{\alpha} \partial^{\gamma} n\right)+\partial^{\gamma} n\left(\Pi^{\alpha \beta}-\frac{\hbar^{2}}{4 m^{2}} \partial^{\alpha} \partial^{\beta} n\right)\right. \\
&+n\left(3 \partial^{\gamma} n \cdot v^{\alpha} v^{\beta}-2 \partial^{\alpha} n \cdot v^{\beta} v^{\gamma}-2 \partial^{\beta} n \cdot v^{\alpha} v^{\gamma}-n v^{\gamma}\left(\partial^{\alpha} v^{\beta}+\partial^{\beta} v^{\alpha}\right)-\partial^{\beta} p^{\alpha \gamma}-\partial^{\alpha} p^{\beta \gamma}\right. \\
&+ \sum_{f} n_{f}\left(\partial^{\gamma} a_{f}^{2}\right) u_{f}^{\alpha} u_{f}^{\beta}+\sum_{f} n_{f} a_{f}^{2}\left(u_{f}^{\beta} \partial^{\alpha} u_{f}^{\gamma}+u_{f}^{\alpha} \partial^{\beta} u_{f}^{\gamma}\right)+\frac{3}{2} v^{\alpha} \sum_{f} n_{f}\left(\partial^{\gamma} a_{f}^{2} \cdot u_{f}^{\beta}-\partial^{\beta} a_{f}^{2} \cdot u_{f}^{\gamma}\right) \\
&\left.\left.+\frac{3}{2} v^{\beta} \sum_{f} n_{f}\left(\partial^{\gamma} a_{f}^{2} \cdot u_{f}^{\alpha}-\partial^{\alpha} a_{f}^{2} \cdot u_{f}^{\gamma}\right)-2 \frac{\hbar^{2}}{m^{2}} \sum_{f} n_{f} \partial^{\gamma} a_{f} \cdot \partial^{\alpha} \partial^{\beta} a_{f}\right)\right] .
\end{aligned}
$$

Equation for the third rank tensor $M^{\alpha \beta \gamma}$ evolution contains the symmetric combination of the third rank force tensors (61) which cam be presented in the following form:

$$
\begin{aligned}
F^{\alpha \beta \gamma}+ & F^{\beta \gamma \alpha}+F^{\gamma \alpha \beta}=-\frac{g}{4}\left[4\left(\Pi_{B}^{\beta \gamma} \partial^{\alpha} n_{B}+\Pi_{B}^{\alpha \gamma} \partial^{\beta} n_{B}+\Pi_{B}^{\alpha \beta} \partial^{\gamma} n_{B}\right)+4\left(\Pi^{\beta \gamma} \partial^{\alpha} n+\Pi^{\alpha \gamma} \partial^{\beta} n+\Pi^{\alpha \beta} \partial^{\gamma} n\right)\right. \\
& +\partial^{\alpha} n\left(3 \Pi^{\beta \gamma}+\frac{\hbar^{2}}{4 m^{2}} \partial^{\beta} \partial^{\gamma} n\right)+\partial^{\beta} n\left(3 \Pi^{\alpha \gamma}+\frac{\hbar^{2}}{4 m^{2}} \partial^{\alpha} \partial^{\gamma} n\right)+\partial^{\gamma} n\left(3 \Pi^{\alpha \beta}+\frac{\hbar^{2}}{4 m^{2}} \partial^{\alpha} \partial^{\beta} n\right)
\end{aligned}
$$




$$
\left.-n\left(\partial^{\alpha} \Pi^{\beta \gamma}+\partial^{\beta} \Pi^{\alpha \gamma}+\partial^{\gamma} \Pi^{\alpha \beta}\right)-\frac{3 \hbar^{2}}{4 m^{2}} n \partial^{\alpha} \partial^{\beta} \partial^{\gamma} n\right] .
$$

The pressure flux evolution equation obtained as the reduction of the third rank tensor $M^{\alpha \beta \gamma}$ evolution equation contains the following combination of the force fields:

$$
\begin{aligned}
F^{\alpha \beta \gamma}+F^{\beta \gamma \alpha}+ & F^{\alpha \beta}-\frac{1}{m n}\left(F^{\alpha} \Pi^{\beta \gamma}+F^{\beta} \Pi^{\alpha \gamma}+F^{\gamma} \Pi^{\alpha \beta}\right)=\frac{g}{4}\left[\partial^{\alpha}\left(n \Pi^{\beta \gamma}\right)+\partial^{\beta}\left(n \Pi^{\alpha \gamma}\right)+\partial^{\gamma}\left(n \Pi^{\alpha \beta}\right)\right]^{\prime} \\
& +\frac{g \hbar^{2}}{16 m^{2}}\left[3 n \partial^{\alpha} \partial^{\beta} \partial^{\gamma} n-\partial^{\alpha} n \cdot \partial^{\beta} \partial^{\gamma} n-\partial^{\beta} n \cdot \partial^{\alpha} \partial^{\gamma} n-\partial^{\gamma} n \cdot \partial^{\alpha} \partial^{\beta} n\right]^{\prime},
\end{aligned}
$$

where symbol []' specifies that product of functions describing the BEC is excluded similarly equations (40) and (41).

The quantum part can be represented

$$
\begin{gathered}
F^{\alpha \beta \gamma}+F^{\beta \gamma \alpha}+F^{\gamma \beta}-\frac{1}{m n}\left(F^{\alpha} \Pi^{\beta \gamma}+F^{\beta} \Pi^{\alpha \gamma}+F^{\gamma} \Pi^{\alpha \beta}\right)=\frac{g}{4}\left[\partial^{\alpha}\left(n \Pi^{\beta \gamma}\right)+\partial^{\beta}\left(n \Pi^{\alpha \gamma}\right)+\partial^{\gamma}\left(n \Pi^{\alpha \beta}\right)\right]^{\prime} \\
\quad+\frac{g \hbar^{2}}{16 m^{2}}\left[\partial^{\alpha}\left(n \partial^{\beta} \partial^{\gamma} n-\partial^{\beta} n \cdot \partial^{\gamma} n\right)+\partial^{\beta}\left(n \partial^{\alpha} \partial^{\gamma} n-\partial^{\alpha} n \cdot \partial^{\gamma} n\right)+\partial^{\gamma}\left(n \partial^{\alpha} \partial^{\beta} n-\partial^{\alpha} n \cdot \partial^{\beta} n\right)\right]^{\prime} .
\end{gathered}
$$

It can be considered as $F^{\alpha \beta \gamma}+F^{\beta \gamma \alpha}+F^{\gamma \alpha \beta}-\frac{1}{m n}\left(F^{\alpha} \Pi^{\beta \gamma}+F^{\beta} \Pi^{\alpha \gamma}+F^{\gamma} \Pi^{\alpha \beta}\right)=\tilde{F}^{\alpha \beta \gamma}+\tilde{F}^{\beta \gamma \alpha}+\tilde{F}^{\gamma \alpha \beta}$, where

$$
\tilde{F}^{\alpha \beta \gamma}=\frac{g}{4}\left[\partial^{\alpha}\left(n \Pi^{\beta \gamma}\right)+\frac{g \hbar^{2}}{16 m^{2}}\left[\partial^{\alpha}\left(n \partial^{\beta} \partial^{\gamma} n-\partial^{\beta} n \cdot \partial^{\gamma} n\right)\right]^{\prime} .\right.
$$

It is the derivative of the second rank tensor.

If we consider the zero temperature limit we find $F^{\alpha \beta \gamma}=\left(-g / 4 m^{3}\right) \Pi_{B}^{\alpha \beta} \partial^{\gamma} n_{B}$. The transition from the equation of evolution for tensor $M^{\alpha \beta \gamma}$ to the equation of evolution for tensor $Q^{\alpha \beta \gamma}$, which is the sibling of $M^{\alpha \beta \gamma}$, but the pressure flux $Q^{\alpha \beta \gamma}$ is defined in the comoving frame leads to the canceling of such term. Hence, the nonzero contribution comes from the quantum part of the third rank force tensor $F_{q f}^{\alpha \beta \gamma}$. The nonzero temperature gives a nonzero contribution of $F^{\alpha \beta \gamma}$ at the transition to the pressure flux evolution equation.

Equation (65) is obtained for all bosons. We need to separate it on the force acting on the BEC and the force acting on the normal fluid.

Finally, we obtain

$$
\begin{gathered}
\tilde{F}_{n}^{\alpha \beta \gamma}+\tilde{F}_{n}^{\beta \gamma \alpha}+\tilde{F}_{n}^{\gamma \alpha \beta}=\frac{g}{4}\left[\partial^{\alpha}\left(n_{n} \Pi_{n}^{\beta \gamma}\right)+\partial^{\beta}\left(n_{n} \Pi_{n}^{\alpha \gamma}\right)+\partial^{\gamma}\left(n_{n} \Pi_{n}^{\alpha \beta}\right)\right. \\
\left.+n_{n} \partial^{\alpha}\left(\Pi_{B}^{\beta \gamma}+\partial^{\beta} \Pi_{B}^{\alpha \gamma}+\partial^{\gamma} \Pi_{B}^{\alpha \beta}\right)+\Pi_{n}^{\beta \gamma} \partial^{\alpha} n_{B}+\Pi_{n}^{\alpha \gamma} \partial^{\beta} n_{B}+\Pi_{n}^{\alpha \beta} \partial^{\gamma} n_{B}\right] \\
+\frac{g \hbar^{2}}{16 m^{2}}\left[3 n_{n} \partial^{\alpha} \partial^{\beta} \partial^{\gamma} n_{n}+3 n_{n} \partial^{\alpha} \partial^{\beta} \partial^{\gamma} n_{B}-\partial^{\alpha} n_{n} \cdot \partial^{\beta} \partial^{\gamma} n_{n}-\partial^{\beta} n_{n} \cdot \partial^{\alpha} \partial^{\gamma} n_{n}-\partial^{\gamma} n_{n} \cdot \partial^{\alpha} \partial^{\beta} n_{n}\right. \\
\left.-\frac{1}{2} \partial^{\alpha} n_{n} \cdot \partial^{\beta} \partial^{\gamma} n_{B}-\frac{1}{2} \partial^{\beta} n_{n} \cdot \partial^{\alpha} \partial^{\gamma} n_{B}-\frac{1}{2} \partial^{\gamma} n_{n} \cdot \partial^{\alpha} \partial^{\beta} n_{B}-\frac{1}{2} \partial^{\alpha} n_{B} \cdot \partial^{\beta} \partial^{\gamma} n_{n}-\frac{1}{2} \partial^{\beta} n_{B} \cdot \partial^{\alpha} \partial^{\gamma} n_{n}-\frac{1}{2} \partial^{\gamma} n_{B} \cdot \partial^{\alpha} \partial^{\beta} n_{n}\right],
\end{gathered}
$$

and

$$
\begin{gathered}
\tilde{F}_{B}^{\alpha \beta \gamma}+\tilde{F}_{B}^{\beta \gamma \alpha}+\tilde{F}_{B}^{\gamma \alpha \beta}=\frac{g}{4}\left[n_{B} \partial^{\alpha}\left(\Pi_{n}^{\beta \gamma}+\partial^{\beta} \Pi_{n}^{\alpha \gamma}+\partial^{\gamma} \Pi_{n}^{\alpha \beta}\right)\right. \\
\left.+\Pi_{B}^{\beta \gamma} \partial^{\alpha} n_{n}+\Pi_{B}^{\alpha \gamma} \partial^{\beta} n_{n}+\Pi_{B}^{\alpha \beta} \partial^{\gamma} n_{n}\right]+\frac{g \hbar^{2}}{16 m^{2}}\left[3 n_{B} \partial^{\alpha} \partial^{\beta} \partial^{\gamma} n_{n}\right. \\
\left.-\frac{1}{2} \partial^{\alpha} n_{B} \cdot \partial^{\beta} \partial^{\gamma} n_{n}-\frac{1}{2} \partial^{\beta} n_{B} \cdot \partial^{\alpha} \partial^{\gamma} n_{n}-\frac{1}{2} \partial^{\gamma} n_{B} \cdot \partial^{\alpha} \partial^{\beta} n_{n}-\frac{1}{2} \partial^{\alpha} n_{n} \cdot \partial^{\beta} \partial^{\gamma} n_{B}-\frac{1}{2} \partial^{\beta} n_{n} \cdot \partial^{\alpha} \partial^{\gamma} n_{B}-\frac{1}{2} \partial^{\gamma} n_{n} \cdot \partial^{\alpha} \partial^{\beta} n_{B}\right] \cdot
\end{gathered}
$$

Equations (66) and (67) gives final expressions for the quasi-classic force fields in the pressure flux evolution equations for two fluid model.

2. The third rank force tensor describing the quantum fluctuation

tribution can be found in the first order by the interac-

The quantum fluctuations in the zero temperature BECs is caused by tensor $F_{q f}^{\alpha \beta \gamma}(26)$. Its major con- 
tion radius approximation. Here, we consider the small nonzero temperature regime of $F_{q f}^{\alpha \beta \gamma}$ for the bosons. So, we obtain its generalization for the two-fluid model. The quantum third rank force tensor $F_{q f}^{\alpha \beta \gamma}(26)$ is calculated in the first order by the interaction radius

$$
\begin{gathered}
F_{q f}^{\alpha \beta \gamma}=-\frac{\hbar^{2}}{8 m^{2}} \partial^{\delta} \int d R \sum_{i, j . i \neq j} \delta\left(\mathbf{r}-\mathbf{R}_{i j}\right) \times \\
\times r_{i j}^{\delta} \partial_{i}^{\alpha} \partial_{i}^{\beta} \partial_{i}^{\gamma} U\left(\mathbf{r}_{i j}\right) \Psi^{*}\left(R^{\prime}, t\right) \Psi\left(R^{\prime}, t\right) .
\end{gathered}
$$

In formula (68) for tensor $F_{q f}^{\alpha \beta \gamma}$ we have separation of the integral containing the interaction potential, as we have at the calculation of other force fields above. However, here we obtain different integral $\int r^{\alpha} \partial^{\beta} \partial^{\gamma} \partial^{\delta}$. Calculation of this integral leads to the second interaction constant given below in the simplified expression for the quantum third rank force tensor

$$
F_{q f}^{\alpha \beta \gamma}=\frac{\hbar^{2}}{8 m^{2}} g_{2} I_{0}^{\alpha \beta \gamma \delta} \partial^{\delta} \operatorname{Trn}_{2}\left(\mathbf{r}, \mathbf{r}^{\prime}, t\right)
$$

where

$$
g_{2}=\frac{2}{3} \int d \mathbf{r} U^{\prime \prime}(r)
$$

and

$$
I_{0}^{\alpha \beta \gamma \delta}=\delta^{\alpha \beta} \delta^{\gamma \delta}+\delta^{\alpha \gamma} \delta^{\beta \delta}+\delta^{\alpha \delta} \delta^{\beta \gamma}
$$

Calculation of the two-particle concentration leads to the following expression:

$$
F_{q f}^{\alpha \beta \gamma}=\frac{\hbar^{2}}{8 m^{2}} g_{2} I_{0}^{\alpha \beta \gamma \delta} \partial^{\delta}\left(2 n_{n}^{2}+4 n_{B} n_{n}+n_{B}^{2}\right)
$$

Here we have $\partial^{\delta}\left(2 n_{n}^{2}+4 n_{B} n_{n}+n_{B}^{2}\right)$. Next, we open brackets and find $\left(4 n_{n} \partial^{\delta} n_{n}+4 n_{n} \partial^{\delta} n_{B}+4 n_{B} \partial^{\delta} n_{n}+\right.$ $\left.2 n_{B} \partial^{\delta} n_{B}\right)$. The source of field is under derivative, while the multiplier in front corresponds to the species under the action of field. Hence the first two terms correspond to the force acting on the normal fluid, while the third and fourth terms correspond to the force acting on the BEC.

Therefore, we can separate expression (72) on two parts corresponding to the BEC and to the normal fluid:

$$
F_{q f, B}^{\alpha \beta \gamma}=\frac{\hbar^{2}}{4 m^{2}} g_{2} I_{0}^{\alpha \beta \gamma \delta}\left(2 n_{B} \partial^{\delta} n_{n}+n_{B} \partial^{\delta} n_{B}\right)
$$

and

$$
F_{q f, n}^{\alpha \beta \gamma}=\frac{\hbar^{2}}{2 m^{2}} g_{2} I_{0}^{\alpha \beta \gamma \delta} \partial^{\delta}\left(n_{n} \partial^{\delta} n_{n}+n_{B} \partial^{\delta} n_{n}\right)
$$

\section{HYDRODYNAMIC EQUATIONS FOR TWO FLUID MODEL OF BOSONS WITH NONZERO TEMPERATURE}

This section provides the final set of equations obtained in this paper. The method of the introduction of the velocity field and corresponding representation of the hydrodynamic equations is not described in this paper. It can be found in number other papers, majority of details are given in Refs. [2], 35].

In this regime we have two continuity equations:

$$
\partial_{t} n_{B}+\nabla \cdot\left(n_{B} \mathbf{v}_{B}\right)=0
$$

and

$$
\partial_{t} n_{n}+\nabla \cdot\left(n_{n} \mathbf{v}_{n}\right)=0
$$

The Euler equation for bosons in the BEC state

$$
\begin{aligned}
& m n_{B}\left(\partial_{t}+\mathbf{v}_{B} \cdot \nabla\right) v_{B}^{\alpha}+\partial_{\beta} T_{B}^{\alpha \beta}+\partial_{\beta} p_{q f}^{\alpha \beta} \\
& +g n_{B} \partial^{\alpha} n_{B}=-n_{B} \partial^{\alpha} V_{e x t}-2 g n_{B} \partial^{\alpha} n_{n},
\end{aligned}
$$

where the quantum Bohm potential is given by equation (12) in the noninteracting limit.

The Euler equation for bosons in the excited states corresponding to the nonzero temperature

$$
\begin{gathered}
m n_{n}\left(\partial_{t}+\mathbf{v}_{n} \cdot \nabla\right) v_{n}^{\alpha}+\partial_{\beta} p_{n, e f f}^{\alpha \beta} \\
+2 g n_{n} \partial^{\alpha} n_{n}=-n_{n} \partial^{\alpha} V_{e x t}-2 g n_{n} \partial^{\alpha} n_{B},
\end{gathered}
$$

where the effective pressure tensor $p_{n, e f f}^{\alpha \beta}=p_{n}^{\alpha \beta}+T_{n}^{\alpha \beta}$.

The effective pressure evolution equation for normal boson fluid is also a part of developed and applied hydrodynamic model

$$
\begin{gathered}
\partial_{t} p_{n, e f f}^{\alpha \beta}+v_{n}^{\gamma} \partial_{\gamma} p_{n, e f f}^{\alpha \beta}+p_{n, e f f}^{\alpha \gamma} \partial_{\gamma} v_{n}^{\beta}+p_{n, e f f}^{\beta \gamma} \partial_{\gamma} v_{n}^{\alpha} \\
+p_{n, e f f}^{\alpha \beta} \partial_{\gamma} v_{n}^{\gamma}+\partial_{\gamma} T_{n}^{\alpha \beta \gamma}+\partial_{\gamma} Q_{n}^{\alpha \beta \gamma}=0
\end{gathered}
$$

Moreover, we have the pressure (the quantum Bohm potential) $p_{B, e f f}^{\alpha \beta}=T_{B}^{\alpha \beta}+p_{q f}^{\alpha \beta}$ evolution equation for the $\mathrm{BEC}$

$$
\begin{gathered}
\partial_{t} p_{B, e f f}^{\alpha \beta}+v_{B}^{\gamma} \partial_{\gamma} p_{B, e f f}^{\alpha \beta}+p_{B, e f f}^{\alpha \gamma} \partial_{\gamma} v_{B}^{\beta}+p_{B, e f f}^{\beta \gamma} \partial_{\gamma} v_{B}^{\alpha} \\
+p_{B, e f f}^{\alpha \beta} \partial_{\gamma} v_{B}^{\gamma}+\partial_{\gamma} T_{B}^{\alpha \beta \gamma}+\partial_{\gamma} Q_{q f}^{\alpha \beta \gamma}=0 .
\end{gathered}
$$

Let us to point out the following property of the quantum Bohm potential that it satisfies the following equation for the arbitrary species $a$

$$
\partial_{t} T_{a}^{\alpha \beta}+v_{a}^{\gamma} \partial_{\gamma} T_{a}^{\alpha \beta}+T_{a}^{\alpha \gamma} \partial_{\gamma} v_{a}^{\beta}+T_{a}^{\beta \gamma} \partial_{\gamma} v_{a}^{\alpha}
$$




$$
+T_{a}^{\alpha \beta} \partial_{\gamma} v_{a}^{\gamma}+\partial_{\gamma} T_{a}^{\alpha \beta \gamma}=0 .
$$

It is expected that approximate form of the quantum Bohm potential (12) satisfies equation (81) existing at the zero interaction. Hence, substitute (12) in equation (81) with the zero right-hand side:

$$
\begin{gathered}
\partial^{\beta} \partial^{\gamma} n_{a} \cdot\left(\partial^{\gamma} v_{a}^{\alpha}-\partial^{\alpha} v_{a}^{\gamma}\right)+\partial^{\alpha} \partial^{\gamma} n_{a} \cdot\left(\partial^{\gamma} v_{a}^{\beta}-\partial^{\beta} v_{a}^{\gamma}\right) \\
+\frac{1}{3} \partial_{\gamma} n_{a} \cdot\left(\partial^{\beta} \partial^{\gamma} v_{a}^{\alpha}+\partial^{\alpha} \partial^{\gamma} v_{a}^{\beta}-\partial^{\alpha} \partial^{\beta} v_{a}^{\gamma}\right) \\
+\frac{1}{3} n_{a}\left[\triangle\left(\partial^{\beta} v_{a}^{\alpha}+\partial^{\alpha} v_{a}^{\beta}\right)-\partial^{\alpha} \partial^{\beta}\left(\nabla \cdot \mathbf{v}_{a}\right)\right]=0
\end{gathered}
$$

where the continuity equation is used for the time derivatives of concentration. Make the condition of the potentiality of the velocity field $\mathbf{v}_{a}=\nabla \phi_{a}$. Include it in equation (82) and find that this equation is satisfied.

Hence, we obtain the simplified form of the pressure evolution equations, where the traditional quantum Bohm potential is extracted:

$$
\begin{aligned}
\partial_{t} p_{q f, B}^{\alpha \beta}+ & v_{B}^{\gamma} \partial_{\gamma} p_{q f, B}^{\alpha \beta}+p_{q f, B}^{\alpha \gamma} \partial_{\gamma} v_{B}^{\beta}+p_{q f, B}^{\beta \gamma} \partial_{\gamma} v_{B}^{\alpha} \\
& +p_{q f, B}^{\alpha \beta} \partial_{\gamma} v_{B}^{\gamma}+\partial_{\gamma} Q_{q f, B}^{\alpha \beta \gamma}=0
\end{aligned}
$$

and

$$
\begin{gathered}
\partial_{t} p_{n}^{\alpha \beta}+v_{n}^{\gamma} \partial_{\gamma} p_{n}^{\alpha \beta}+p_{n}^{\alpha \gamma} \partial_{\gamma} v_{n}^{\beta}+p_{n}^{\beta \gamma} \partial_{\gamma} v_{n}^{\alpha} \\
+p_{n}^{\alpha \beta} \partial_{\gamma} v_{n}^{\gamma}+\partial_{\gamma} Q_{n}^{\alpha \beta \gamma}=0 .
\end{gathered}
$$

Equation for the evolution of quantum-thermal part of the third rank tensor is [4], 43]:

$$
\begin{gathered}
\partial_{t} Q_{q f}^{\alpha \beta \gamma}+\partial_{\delta}\left(v_{B}^{\delta} Q_{q f}^{\alpha \beta \gamma}\right)+Q_{q f}^{\alpha \gamma \delta} \partial_{\delta} v_{B}^{\beta}+Q_{q f}^{\beta \gamma \delta} \partial_{\delta} v_{B}^{\alpha}+Q_{q f}^{\alpha \beta \delta} \partial_{\delta} v_{B}^{\gamma} \\
\left.=\frac{\hbar^{2}}{4 m^{2}} g_{2} I_{0}^{\alpha \beta \gamma \delta}\left(n_{B} \partial^{\delta} n_{B}+2 n_{B} \partial^{\delta} n_{n}\right)\right) \\
-\frac{1}{m} n_{B} \partial_{\alpha} \partial_{\beta} \partial_{\gamma} V_{e x t}+\tilde{F}_{B}^{\alpha \beta \gamma}+\tilde{F}_{B}^{\beta \gamma \alpha}+\tilde{F}_{B}^{\gamma \alpha \beta}
\end{gathered}
$$$$
+\frac{1}{m n}\left(p_{q f, e f f}^{\alpha \beta} \partial^{\delta} p_{q f, e f f}^{\gamma \delta}+p_{q f, e f f}^{\alpha \gamma} \partial^{\delta} p_{q f, e f f}^{\beta \delta}+p_{q f, e f f}^{\beta \gamma} \partial^{\delta} p_{q f, e f f}^{\alpha \delta}\right),
$$

and

$$
\begin{gathered}
\partial_{t} Q_{n}^{\alpha \beta \gamma}+\partial_{\delta}\left(v_{n}^{\delta} Q_{n}^{\alpha \beta \gamma}\right)+Q_{n}^{\alpha \gamma \delta} \partial_{\delta} v_{n}^{\beta}+Q_{n}^{\beta \gamma \delta} \partial_{\delta} v_{n}^{\alpha}+Q_{n}^{\alpha \beta \delta} \partial_{\delta} v_{n}^{\gamma} \\
=\frac{\hbar^{2}}{2 m^{2}} g_{2} I_{0}^{\alpha \beta \gamma \delta}\left(n_{n} \partial^{\delta} n_{n}+n_{n} \partial^{\delta} n_{B}\right)
\end{gathered}
$$

$$
-\frac{1}{m} n_{n} \partial_{\alpha} \partial_{\beta} \partial_{\gamma} V_{e x t}+\tilde{F}_{n}^{\alpha \beta \gamma}+\tilde{F}_{n}^{\beta \gamma \alpha}+\tilde{F}_{n}^{\gamma \alpha \beta}
$$

$+\frac{1}{m n}\left(p_{n, e f f}^{\alpha \beta} \partial^{\delta} p_{n, e f f}^{\gamma \delta}+p_{n, e f f}^{\alpha \gamma} \partial^{\delta} p_{n, e f f}^{\beta \delta}+p_{n, e f f}^{\beta \gamma} \partial^{\delta} p_{n, e f f}^{\alpha \delta}\right)$,

where $\tilde{F}_{a}^{\alpha \beta \gamma}$ is not presented explicitly since equations (66) and (67) show that required expressions are rather large.

Refs. 2], 3]. Hydrodynamic model for fermions with pressure evolution is derived in Refs. [35], [46], [47].

Terms proportional to $p_{a, e f f}^{\alpha \beta} \partial^{\delta} p_{a, e f f}^{\gamma \delta}$ appears in the pressure flux evolution equation, but it leads to the contribution beyond the chosen approximation [48], [49].

Term containing the external potential $-\frac{1}{m} n_{a} \partial_{\alpha} \partial_{\beta} \partial_{\gamma} V_{\text {ext }}$ goes to zero for the parabolic trap. However, it can give some nontrivial contribution for other form of potentials.

\section{BEC DYNAMICS UNDER THE INFLUENCE OF THE QUANTUM FLUCTUATIONS}

Developed model shows that there is nontrivial evolution equation for the pressure and the pressure flux of the BEC. Therefore, the well-known model of BEC is extended in spite the fact that the kinetic pressure tensor is expected to be equal to zero due to zero temperature. However, the quantum fluctuations lead to the nonzero occupation numbers for the excited states.

If we need to consider pure BEC we need to drop the contribution of the normal fluid in the model presented above. Therefore, let us summarize the BEC model in parabolic traps:

$$
\begin{gathered}
\partial_{t} n_{B}+\nabla \cdot\left(n_{B} \mathbf{v}_{B}\right)=0, \\
m n_{B}\left(\partial_{t}+\mathbf{v}_{B} \cdot \nabla\right) v_{B}^{\alpha}+\partial_{\beta}\left(p_{q f}^{\alpha \beta}+T_{B}^{\alpha \beta}\right) \\
+g n_{B} \partial^{\alpha} n_{B}+n_{B} \partial^{\alpha} V_{e x t}=0, \\
\partial_{t} p_{q f}^{\alpha \beta}+v_{B}^{\gamma} \partial_{\gamma} p_{q f}^{\alpha \beta}+p_{q f}^{\alpha \gamma} \partial_{\gamma} v_{B}^{\beta}+p_{q f}^{\beta \gamma} \partial_{\gamma} v_{B}^{\alpha} \\
+p_{q f}^{\alpha \beta} \partial_{\gamma} v_{B}^{\gamma}+\partial_{\gamma} Q_{q f}^{\alpha \beta \gamma}=0,
\end{gathered}
$$

and

$$
\begin{gathered}
\partial_{t} Q_{q f}^{\alpha \beta \gamma}+\partial_{\delta}\left(v^{\delta} Q_{q f}^{\alpha \beta \gamma}\right)+Q_{q f}^{\alpha \gamma \delta} \partial_{\delta} v^{\beta} \\
+Q_{q f}^{\beta \gamma \delta} \partial_{\delta} v^{\alpha}+Q_{q f}^{\alpha \beta \delta} \partial_{\delta} v^{\gamma}=\frac{\hbar^{2}}{4 m^{2}} g_{2} I_{0}^{\alpha \beta \gamma \delta} n \partial^{\delta} n .
\end{gathered}
$$

This simplified model is reported earlier in Refs. [4], [43], [44], where the dipole-dipole interaction is also considered. It has been demonstrated that the quantum fluctuations gives mechanisms for the instability of the small 
amplitude perturbations [4]. Moreover, the dipolar part of the quantum fluctuations creates conditions for the bright soliton in the repulsive BECs [44]. The developed in previous section model provides proper generalization of earlier model giving the small temperature contribution.

\section{CONCLUSION}

Revision of the two-fluid model for the finite temperature ultracold bosons has been presented through the derivation of the number of particles, the momentum, the momentum flux, and the third rank tensor balance equations. The derivation has been based on the trace of the microscopic dynamics of quantum particles via the application of the many-particle microscopic Schrodinger equation. Hence, the microscopic Schrodinger equation determines the time evolution for the macroscopic functions describing the collective motion of bosons.

General equations have been derived for the arbitrary strength of interaction and the arbitrary temperatures. The set of equations has been restricted by the third rank kinematic tensor (the flux of pressure). The truncation is made for the low temperatures weakly interacting bosons after the derivation of the general structure of hydrodynamic equations. Therefore, the thermal part of the fourth rank kinematic tensor has been taken equal to zero. Next, the terms containing the interaction potential have been considered for the short-range interaction. The small radius of interaction provides the small parameter for the expansion. The expansion is made in the force field in the Euler equation, the force tensor field in the momentum flux equation, and the third rank force tensor in the pressure flux evolution equation. The first term in expansion on the small interparticle distance has been considered in each expansion, which corresponds to the first order by the interaction radius.

This model allows to gain the quantum fluctuations which is the essential property of the BECs. Moreover, the interaction causing the quantum fluctuations has been consider at the finite temperature.

The functions obtained in the first order by the interaction radius have been expressed via the trace of twoparticle functions. The two-particle functions have been calculated for the weakly interacting bosons.

The single species of 0 -spin bosons has been considered. Therefore, the single fluid hydrodynamics has been derived. Next, it has been included that the concentration of particles, the current of particles (the momentum density), the momentum flux, and the current of the momentum flux are additive functions. Consequently, they can be easily splitted on two parts: the BEC and the normal fluid of bosons (the non BEC part). Hence, the two fluid model of single species of bosons is obtained. This separation on two fluids has been made in general form of equations.

\section{ACKNOWLEDGEMENTS}

Work is supported by the Russian Foundation for Basic Research (grant no. 20-02-00476). This paper has been supported by the RUDN University Strategic Academic Leadership Program.
[1] F. Dalfovo, S. Giorgini, L. P. Pitaevskii, and S. Stringari, Rev. Mod. Phys. 71, 463 (1999).

[2] P. A. Andreev, L. S. Kuz'menkov, Phys. Rev. A 78, 053624 (2008).

[3] P. A. Andreev, Laser Phys. 29, 035502 (2019).

[4] P. A. Andreev, arXiv:2005.13503 (accepted to Chaos).

[5] T. D. Lee, K. Huang, and C. N. Yang, Phys. Rev. 106, 1135 (1957).

[6] L. Pitaevskii and S. Stringari, Phys. Rev. Lett. 81, 4541 (1998).

[7] E. Braaten and J. Pearson, Phys. Rev. Lett. 82, 255 (1999).

[8] G. E. Astrakharchik, R. Combescot, X. Leyronas, and S. Stringari, Phys. Rev. Lett. 95, 030404 (2005).

[9] K. Xu, Y. Liu, D. E. Miller, J. K. Chin, W. Setiawan, and W. Ketterle, Phys. Rev. Lett. 96, 180405 (2006).

[10] A. Altmeyer, S. Riedl, C. Kohstall, M. J. Wright, R. Geursen, M. Bartenstein, C. Chin, J. Hecker Denschlag, and R. Grimm, Phys. Rev. Lett. 98, 040401 (2007).

[11] S. B. Papp, J. M. Pino, R. J. Wild, S. Ronen, C. E. Wieman, D. S. Jin, and E. A. Cornell, Phys. Rev. Lett. 101, 135301 (2008).

[12] H. Kadau, M. Schmitt, M. Wenzel, C. Wink, T. Maier, I. Ferrier-Barbut, T. Pfau, Nature 530, 194 (2016).
[13] I. Ferrier-Barbut, H. Kadau, M. Schmitt, M. Wenzel, and T. Pfau, Phys. Rev. Lett. 116, 215301 (2016).

[14] D. Baillie, R. M. Wilson, R. N. Bisset, and P. B. Blakie, Phys. Rev. A 94, 021602(R) (2016).

[15] R. N. Bisset, R. M. Wilson, D. Baillie, P. B. Blakie, Phys. Rev. A 94, 033619 (2016).

[16] F. Wachtler and L. Santos, Phys. Rev. A 93, 061603R (2016).

[17] F. Wachtler and L. Santos, Phys. Rev. A 94, 043618 (2016).

[18] P. B. Blakie, Phys. Rev. A 93, 033644 (2016).

[19] A. Boudjemaa and N. Guebli, Phys. Rev. A 102, 023302 (2020).

[20] V. Heinonen, K. J. Burns, and J. Dunkel, Phys. Rev. A 99, 063621 (2019).

[21] B. A. Malomed, Physica D 399, 108 (2019).

[22] E. Shamriz, Z. Chen, and B. A. Malomed, Phys. Rev. A 101, 063628 (2020).

[23] Z. Li, J.-S. Pan, and W. Vincent Liu, Phys. Rev. A 100, 053620 (2019).

[24] E. Aybar and M. O. Oktel, Phys. Rev. A 99, 013620 (2019).

[25] P. Examilioti, and G. M. Kavoulakis, J. Phys. B: At. Mol. Opt. Phys. 53, 175301 (2020). 
[26] T. Miyakawa, S. Nakamura, H. Yabu, Phys. Rev. A 101, 033613 (2020).

[27] F. Bottcher, Jan-Niklas Schmidt, J. Hertkorn, Kevin S. H. Ng, Sean D. Graham, M. Guo, T. Langen, and T. Pfau, arXiv:2007.06391.

[28] R. N. Bisset, L. A. P. Ardila, and L. Santos, Phys. Rev. Lett. 126, 025301 (2021).

[29] Y. Wang, L. Guo, S. Yi, and T. Shi, Phys. Rev. Research 2, 043074 (2020).

[30] M. J. Edmonds, T. Bland, and N. G. Parker, J. Phys. Commun. 4, 125008 (2020)

[31] D. Baillie and P. B. Blakie, Phys. Rev. A 101, 043606 (2020).

[32] A. Griffin, Phys. Rev. B 53, 9341 (1996).

[33] L. S. Kuz'menkov, S. G. Maksimov, and V. V. Fedoseev, Theor. Math. Fiz. 126, 136 (2001) [Theoretical and Mathematical Physics 126, 110 (2001)].

[34] P. A. Andreev, L. S. Kuz'menkov, Prog. Theor. Exp. Phys. 2019, 053J01 (2019).

[35] P. A. Andreev, arXiv:2001.02764

[36] J. W. M. Bush, Y. Couder, T. Gilet, P. A. Milewski, and A. Nachbin, Chaos 28, 096001 (2018).
[37] Y. Couder, S. Protiere, E. Fort, and A. Boudaoud, Nature 437, 208 (2005).

[38] J. W. M. Bush, Annu. Rev. Fluid Mech. 47, 269-292 (2015).

[39] T. Cristea-Platon, P. J. Saenz, and J. W. M. Bush, Chaos 28, 096116 (2018).

[40] A. Chowdury, A. Ankiewicz, N. Akhmediev, and W. Chang, Chaos 28, 123116 (2018).

[41] N. B. Budanur, and M. Fleury, Chaos 29, 013122 (2019).

[42] P. A. Andreev, Int. J. Mod. Phys. B 27, 1350017 (2013).

[43] P. A. Andreev, arXiv:2007.15045

[44] P. A. Andreev, arXiv:2009.12720

[45] G. M. Kavoulakis, C. J. Pethick, and H. Smith, Phys. Rev. A 57, 2938 (1998).

[46] P. A. Andreev, arXiv:1912.00843.

[47] P. A. Andreev, K. V. Antipin, M. I. Trukhanova, Laser Phys. 31, 015501 (2021).

[48] I. Tokatly, O. Pankratov, Phys. Rev. B 60, 15550 (1999).

[49] I. V. Tokatly, O. Pankratov, Phys. Rev. B 62, 2759 (2000). 